\title{
Quantum critical transport, duality, and M theory
}

\section{Citation}

Herzog, Christopher P., Pavel Kovtun, Subir Sachdev, and Dam Thanh Son. 2007. “Quantum Critical Transport, Duality, and M Theory." Physical Review D 75 (8). https://doi.org/10.1103/ physrevd.75.085020.

\section{Permanent link}

http://nrs.harvard.edu/urn-3:HUL.InstRepos:41417263

\section{Terms of Use}

This article was downloaded from Harvard University's DASH repository, and is made available under the terms and conditions applicable to Other Posted Material, as set forth at http:// nrs.harvard.edu/urn-3:HUL.InstRepos:dash.current.terms-of-use\#LAA

\section{Share Your Story}

The Harvard community has made this article openly available.

Please share how this access benefits you. Submit a story.

\section{Accessibility}


hep-th/0701036

NSF-KITP-06-129

INT-PUB 07-01

\title{
Quantum critical transport, duality, and M-theory
}

\author{
Christopher P. Herzog, ${ }^{1}$ Pavel Kovtun, ${ }^{2}$ Subir Sachdev, ${ }^{3}$ and Dam Thanh Son ${ }^{4}$ \\ ${ }^{1}$ Department of Physics, University of Washington, Seattle, WA 98195-1560, USA \\ ${ }^{2}$ Kavli Institute for Theoretical Physics, \\ University of California, Santa Barbara, CA 93106-4030, USA \\ ${ }^{3}$ Department of Physics, Harvard University, Cambridge MA 02138, USA \\ ${ }^{4}$ Institute for Nuclear Theory, University of Washington, Seattle, WA 98195-1550, USA
}

(Dated: March 2007)

\begin{abstract}
We consider charge transport properties of $2+1$ dimensional conformal field theories at non-zero temperature. For theories with only Abelian U(1) charges, we describe the action of particle-vortex duality on the hydrodynamic-to-collisionless crossover function: this leads to powerful functional constraints for self-dual theories. For $\mathcal{N}=8$ supersymmetric, $\mathrm{SU}(N)$ Yang-Mills theory at the conformal fixed point, exact hydrodynamic-to-collisionless crossover functions of the $\mathrm{SO}(8) \mathrm{R}$ currents can be obtained in the large $N$ limit by applying the AdS/CFT correspondence to Mtheory. In the gravity theory, fluctuating currents are mapped to fluctuating gauge fields in the background of a black hole in 3+1 dimensional anti-de Sitter space. The electromagnetic self-duality of the $3+1$ dimensional theory implies that the correlators of the R-currents obey a functional constraint similar to that found from particle-vortex duality in $2+1$ dimensional Abelian theories. Thus the 2+1 dimensional, superconformal Yang Mills theory obeys a "holographic self duality" in the large $N$ limit, and perhaps more generally.
\end{abstract}




\section{INTRODUCTION}

The quantum phase transitions of two (spatial) dimensional systems have been the focus of much study in the condensed matter community. Prominent examples include the superfluid-insulator transition in thin films [1-3], the transitions between various quantum Hall states $[4,5]$, and magnetic ordering transitions of Mott insulators and superconductors which have applications to the cuprate compounds [6-8]. Of particular interest in this paper are the transport properties of conserved quantities such as the electrical charge or the total spin: these are characterized by a (charge or spin) conductivity $\sigma$, which can in general be a complicated function of frequency $\omega$, wavevector $k$, temperature $T$, and various couplings characterizing the ground state.

It is often the case that the quantum critical point is described by a strongly interacting quantum field theory in $2+1$ spacetime dimensions $D$. Examples are $(i)$ the superfluidinsulator transition in the boson Hubbard model at integer filling [9-11], which is described by the $\varphi^{4}$ field theory with $\mathrm{O}(2)$ symmetry, and so is controlled by the Wilson-Fisher fixed point in $D=2+1$; $(i i)$ the spin-gap paramagnet to Néel order transition of coupled spin dimers/ladders/layers which is described by the $\mathrm{O}(3) \varphi^{4}$ field theory $[12,13]$; and (iii) the 'deconfined' critical point of a $S=1 / 2$ antiferromagnet between a Néel and a valence bond solid state $[14,15]$, which is described by the $\mathbb{C P}^{1}$ model with a non-compact $U(1)$ gauge field [16]. In all these cases the critical point is described by a relativistic conformal field theory (CFT). With an eye towards such experimentally motivated applications, our purpose here is to explore the transport properties of general interacting CFTs in $D=2+1$.

A crucial property of CFTs in $D=2+1$ (which actually applies more generally to any critical theory in 2 spatial dimensions which obeys hyperscaling) is that the conductivity is $1 / \hbar$ times a dimensionless number. For U(1) currents, there is also a prefactor of $\left(e^{*}\right)^{2}$ where $e^{*}$ is the unit of charge - we will drop this factor below. For non-Abelian Noether currents, the normalization of charge is set by a conventional normalization of the generators of the Lie algebra. We will be working with relativistic theories, and therefore set $\hbar=k_{B}=c=1$.

Initial discussions [17-19] of this dimensionless conductivity at the quantum critical point were expressed in terms of ground state correlations of the CFT. Let $J_{\mu}^{a}$ represent the set of conserved currents of the theory; here $\mu=0,1,2$ is a spacetime index, and $a$ labels the generators of the global symmetry. In the CFT, $J_{\mu}^{a}(x)$ has dimension 2 , and so current conservation combined with Lorentz and scale invariance imply for the Fourier transform of the retarded correlator $C_{\mu \nu}^{a b}(x)$ at zero temperature ${ }^{1}$ :

$$
\left.C_{\mu \nu}^{a b}(p)\right|_{T=0}=\sqrt{p^{2}}\left(\eta_{\mu \nu}-\frac{p_{\mu} p_{\nu}}{p^{2}}\right) K_{a b},
$$

\footnotetext{
1 If needed, a "diamagnetic" or "contact" term has been subtracted to ensure current conservation. In theories with Chern-Simons terms, an additional term proportional to $\epsilon_{\mu \nu \lambda} p_{\lambda}$ is permitted in Eq. (1.1) and the $T>0$ generalization in Eq. (1.4). See Appendix B.
} 
where $\eta_{\mu \nu}=\operatorname{diag}(-1,1,1), p_{\mu}=(-\omega, \boldsymbol{k})$ is spacetime momentum, and $p^{2}=\boldsymbol{k}^{2}-\omega^{2}$. We define $\sqrt{p^{2}}$ so that it is analytic in the upper-half-plane of $\omega$ and $\operatorname{Im} \sqrt{p^{2}} \leq 0$ for $\omega>0$. The parameters $K_{a b}$ are a set of universal, momentum-independent dimensionless constants characterizing the CFT, which are the analog of the central charge of the Kac-Moody algebra of CFTs in $D=1+1$. Application of the Kubo formula at $T=0$ shows that $[17,19]$ the $K_{a b}$ are equal to the conductivities $\sigma_{a b}=K_{a b}$, thus setting up the possibility of observing these in experiments.

It was also noted $[18,19]$ that particle-vortex duality [20-22] of theories with Abelian symmetry mapped the $T=0$ conductivities to their inverse (we review this mapping in Section II). In self-dual theories, this imposes constraints on the values of the $K_{a b}$, possibly allowing them to be determined exactly. However, the field theories considered in these early works were not self-dual (see Appendix B). Duality, and possible self-duality, was also considered in the context of theories containing Chern-Simons terms, relevant to quantum Hall systems [23-28]. We comment on these works in Appendix B, but the body of the paper considers only theories without Chern-Simons terms. For our purposes, more relevant is the self-dual field theory proposed recently by Motrunich and Vishwanath [16], and we discuss its charge transport properties below.

It was subsequently pointed out [29-31] that the $K_{a b}$ are not the d.c. conductivities observed at small but non-zero temperature. The key point [31-33] is that at non-zero $T$, the time $1 / T$ is a characteristic 'collision' or 'decoherence' time of the excitations of the CFT. Consequently the transport at $\omega \ll T$ obeys 'collision-dominated' hydrodynamics, while that at $\omega \gg T$ involves 'collisionless' motion of excitations above the ground state. Therefore, the limits $\omega \rightarrow 0$ and $T \rightarrow 0$ do not, in general, commute, and must be taken with great care; the constants $K_{a b}$ above are computed in the limit $\omega / T \rightarrow \infty$, while the d.c. conductivities involve $\omega / T \rightarrow 0$.

This contrast between the collisionless and collision-dominated behavior is most clearly displayed in the correlations of the conserved densities. Taking the $t$ component of Eq. (1.1) we obtain the response

$$
C_{t t}^{a b}(\omega, k)=K_{a b} \frac{-k^{2}}{\sqrt{k^{2}-\omega^{2}}} \quad, \quad|| \omega|-k| \gg T,
$$

which characterizes the 'collisionless' response of the CFT at $T=0$. We have also noted above that we expect the same result to apply at $T>0$ provided $\omega$ and $k=|\boldsymbol{k}|$ are large enough, and away from the light cone. The $T>0$ correlations are the Fourier transform of the retarded real time correlators. These are related by analytic continuation to the Euclidean space correlations defined at the Matsubara frequencies, which are integer multiples of $2 \pi T$. The low frequency hydrodynamic regime $\omega \ll T$ is only defined in real time (Minkowski space). In this regime, the arguments of Ref. [29] imply that the 'collision- 
dominated' response has the structure

$$
C_{t t}^{a b}(\omega, k)=\sum_{\lambda} \chi_{a b}^{\lambda} \frac{-D_{\lambda} k^{2}}{-i \omega+D_{\lambda} k^{2}} \quad, \quad|\omega|, k \ll T,
$$

where $D_{\lambda}$ are the diffusion constants of a set of diffusive eigenmodes labelled by $\lambda$, and $\chi_{a b}^{\lambda}$ are the corresponding susceptibilities. Scaling arguments imply that [34] $D_{\lambda}=\mathcal{D}_{\lambda} / T$ and $\chi_{a b}^{\lambda}=\mathcal{C}_{a b}^{\lambda} T$, where the $\mathcal{D}_{\lambda}, \mathcal{C}_{a b}^{\lambda}$ are a set of universal numbers characterizing the hydrodynamic response of the CFT. The d.c. conductivities can be obtained from the Kubo formula by $\sigma_{a b}=\lim _{\omega \rightarrow 0} \lim _{k \rightarrow 0}\left(i \omega / k^{2}\right) C_{t t}^{a b}$, where the order of limits is significant. At any fixed $T>0$, the limits of small $k$ and $\omega$ imply that this Kubo formula has to be applied to Eq. (1.3), and leads to Einstein relations between the $T$-independent universal conductivities and the diffusivities. The distinct forms of Eqs. (1.2) and (1.3) make it clear that, in general, the universal d.c. conductivities bear no direct relationship to the $K_{a b}$; the latter, as we will see below in Eq. (1.7), are related to the high frequency conductivity.

It is worth noting here in passing that the structure in Eq. (1.3) does not apply to CFTs in $D=1+1$, where a result analogous to Eq. (1.2) holds also in the low frequency and low momentum limit; see Appendix A for further discussion of this important point.

Returning to consideration of all the components of the $C_{\mu \nu}^{a b}$ in $D=2+1$, an alternative presentation of the collisionless-to-hydrodynamic crossover is obtained by writing down the generalization of Eq. (1.1) to $T>0$. Current conservation and spatial rotational invariance, without Lorentz invariance at $T>0$, generalize Eq. (1.1) to

$$
C_{\mu \nu}^{a b}(\omega, \boldsymbol{k})=\sqrt{p^{2}}\left(P_{\mu \nu}^{T} K_{a b}^{T}(\omega, k)+P_{\mu \nu}^{L} K_{a b}^{L}(\omega, k)\right)
$$

where $k=|\boldsymbol{k}|$, and $P_{\mu \nu}^{T}$ and $P_{\mu \nu}^{L}$ are orthogonal projectors defined by

$$
P_{00}^{T}=P_{0 i}^{T}=P_{i 0}^{T}=0 \quad, \quad P_{i j}^{T}=\delta_{i j}-\frac{k_{i} k_{j}}{k^{2}}, \quad P_{\mu \nu}^{L}=\left(\eta_{\mu \nu}-\frac{p_{\mu} p_{\nu}}{p^{2}}\right)-P_{\mu \nu}^{T},
$$

with the indices $i, j$ running over the 2 spatial components. The constants $K_{a b}$ have each been replaced by two dimensionless, universal, temperature-dependent functions $K_{a b}^{L, T}(\omega, k)$, characterizing the longitudinal and transverse response. These functions are dimensionless, and hence they can only depend upon the dimensionless ratios $\omega / T$ and $k / T$, as is also the case for the conductivities. Spatial rotational invariance, and the existence of finite correlation length at $T>0$ which ensures analyticity at small $\boldsymbol{k}$, imply that the longitudinal and transverse response are equal to each other at $\boldsymbol{k}=0$, and, by the Kubo formula, are both equal to the zero momentum, frequency dependent complex conductivity, $\sigma_{a b}(\omega / T)$ :

$$
\sigma_{a b}(\omega / T)=K_{a b}^{L}(\omega, 0)=K_{a b}^{T}(\omega, 0) .
$$

Also at $T=0$, these functions reduce to the constants in Eq. (1.1):

$$
\sigma_{a b}(\infty)=K_{a b}=\left.K_{a b}^{L}(\omega, k)\right|_{T=0}=\left.K_{a b}^{T}(\omega, k)\right|_{T=0} .
$$


The functions $K_{a b}^{L, T}(\omega, k)$ are clearly of great physical interest, and it would be useful to compute them for a variety of CFTs. A number of computations have appeared [29, 30, 3538], and show interesting structure in the conductivity as a function of $\omega / T$, encoding the hydrodynamic-to-collisionless crossover for a variety of tractable models. Here we will present some additional results which shed light on the role duality can play on the form of these functions.

In Section II we will consider the role of duality in Abelian systems, by examining the selfdual non-compact, easy-plane, $\mathbb{C P}^{1}$ field theory discussed by Motrunich and Vishwanath [16]. Closely related results apply to other Abelian CFTs whose particle-vortex duals have been described in the literature [28, 39-42], some of which are supersymmetric (in which case, particle-vortex duality is known as 'mirror symmetry'). The Lagrangian formulation of the $\mathbb{C P}^{1}$ theory involves two complex scalar fields and one gauge field $A_{\mu}$, which is coupled to a gauge current $J_{1 \mu}$. The theory has a global $\mathrm{U}(1) \times Z_{2}$ symmetry, and we will denote by $J_{2 \mu}$ the Noether current arising from the U(1) global symmetry. There is another conserved current, the topological current $J_{\text {top }}^{\mu}=\epsilon^{\mu \nu \lambda} \partial_{\nu} A_{\lambda}$, which is conserved by the Bianchi identity. The topological and Noether currents exchange under the self-duality. As we will see in Section II, the two-point correlator of $J_{\text {top }}^{\mu}$ is the inverse of that of $J_{1 \mu}$. We use the notations of Eqs. (1.1), (1.4) with $a, b=1,2$.

The $Z_{2}$ symmetry ensures that the cross-correlations of the $J_{1 \mu}, J_{2 \mu}$ currents vanish, and consequently there are only two constants $K_{1} \equiv K_{11}$ and $K_{2} \equiv K_{22}$ in Eq. (1.1), and similarly for the $T>0$ functions in Eq. (1.4). We examine the duality transformations of these function in Section II and show that the existence of a self-dual critical point leads to the functional relations ${ }^{2}$

$$
\begin{aligned}
& K_{1}^{L}(\omega, k) K_{2}^{T}(\omega, k)=\frac{1}{\pi^{2}}, \\
& K_{2}^{L}(\omega, k) K_{1}^{T}(\omega, k)=\frac{1}{\pi^{2}},
\end{aligned}
$$

which hold for general $T$, while for the constants in Eq. (1.1) this implies $K_{1} K_{2}=1 / \pi^{2}$. Note that these relations are not sufficient to determine the conductivities $\sigma_{1,2}(\omega / T)$; from Eq. (1.6), only their product obeys $\sigma_{1}(\omega / T) \sigma_{2}(\omega / T)=1 / \pi^{2}$, at all $\omega / T$. Thus we expect that for this self-dual model, the conductivities will remain non-trivial functions of $\omega / T$ exhibiting the hydrodynamic-collisionless crossover, and their functional form has to be determined from the solution of a quantum Boltzmann equation.

In Section III, we turn to a field theory with non-Abelian symmetries: the supersymmetric Yang Mills (SYM) gauge theory with a $\mathrm{SU}(N)$ gauge group and $\mathcal{N}=8$ supersymmetry [43]. At long distances, the theory flows under the renormalization group to a strongly coupled $2+1$ dimensional $\mathcal{N}=8$ superconformal field theory (SCFT), which is believed to describe

\footnotetext{
${ }^{2}$ We only keep the one-photon irreducible (1PI) part in $K_{1}^{L, T}$, as explained in Section II.
} 
degrees of freedom on a stack of $N$ M2-branes $[44,45]$. In the limit of large $N$, the SCFT can be analyzed by using the AdS/CFT correspondence [46]. The gravity description of the SCFT is given by M-theory on $3+1$ dimensional anti-de Sitter space times a seven-sphere, and in the large $N$ limit corresponds to $10+1$ dimensional supergravity on $\mathrm{AdS}_{4} \times S^{7}$. The AdS/CFT correspondence provides a method to compute real-time response functions at finite temperature [47, 48], in which case the gravity theory contains a black hole in $\mathrm{AdS}_{4}$. In the limit of low frequency and momentum $\omega \ll T, k \ll T$ one finds hydrodynamic behavior in the SCFT [49]. ${ }^{3}$ The surprising solvability in this limit therefore demands our attention. ${ }^{4}$

The $2+1$ dimensional SCFT has a global SO(8) R-symmetry (the symmetry of the sevensphere in the supergravity description), and therefore has a set of conserved currents $J_{\mu}^{a}$, $a=1, \ldots, 28$. The $\mathrm{SO}(8)$ symmetry implies that $K_{a b}=K \delta_{a b}$, and so there is only a single universal constant $K$ at zero temperature. Similarly, in Eq. (1.4) there are only two independent functions $K^{L}(\omega, k)$ and $K^{T}(\omega, k)$ which characterize the CFT response at finite temperature. In Section III we will compute these functions in the $N \rightarrow \infty$ limit, for all values of $\omega / T$ and $k / T$. We also prove that these functions obey the identity

$$
K^{L}(\omega, k) K^{T}(\omega, k)=\frac{N^{3}}{18 \pi^{2}},
$$

at general $T$, which is strikingly similar to Eqs. (1.8). Now this relation and Eq. (1.6) do indeed determine $\sigma(\omega / T)$ (and $K$ ) to be the frequency-independent constant which is the square root of the right-hand-side of Eq. (1.9). In other words, for this model, the hydrodynamic and high-frequency collisionless conductivities are equal to each other. Nevertheless, the theory does have a hydrodynamic-to-collisionless crossover at all nonzero $k$ (as we will review in Section III), where $K^{L}(\omega, k) \neq K^{T}(\omega, k)$, and so Eq. (1.9) is not sufficient to fix the correlators at $k \neq 0$. Thus the identity Eq. (1.9) causes all signals of the hydrodynamiccollisionless crossover to disappear only at $k=0$.

The similarity of Eq. (1.9) to Eq. (1.8) suggests that explanation of the frequency independence of the conductivity of the $\mathcal{N}=8$ SYM SCFT lies in a self-duality property.

\footnotetext{
${ }^{3}$ Hydrodynamic charge transport at small $\omega$ and $k$ is of course not specific to the $\mathcal{N}=8$ SCFT in $2+1$ dimensions. Hydrodynamics from the supergravity description was first found in strongly coupled $\mathcal{N}=4$ SYM in 3+1 dimensions [50], and later in a variety of other strongly coupled field theories [51-54]. In strongly coupled $\mathcal{N}=4 \mathrm{SYM}$ in $D=3+1$, hydrodynamic to collisionless crossover functions $K^{L, T}(\omega, k)$ were computed in [55]. Note that in $D=3+1$ the conductivity is not dimensionless [31], but is proportional to $T$ in the hydrodynamic limit $\omega \ll T$.

${ }^{4}$ Of course, there are other well-known $D=2+1$ CFTs which are solvable in the large $N$ limit, such as the $O(N) \varphi^{4}$ field theory. However, all of these are theories of particles which are infinitely longlived at $N=\infty$, and so do not exhibit hydrodynamic behavior in this limit. Indeed, an infinite-order resummation of the $1 / N$ expansion is invariably necessary [31] (via the quantum Boltzmann equation) to obtain hydrodynamics. These solvable theories become weakly coupled as $N \rightarrow \infty$, while the $\mathcal{N}=8 \mathrm{SYM}$ remains strongly coupled even as $N \rightarrow \infty$.
} 
Section III demonstrates that this is indeed the case. Under the AdS/CFT correspondence, the two-point correlation function of the $\mathrm{SO}(8)$ R-currents in $D=2+1$ is holographically equivalent to the correlator of a $\mathrm{SO}(8)$ gauge field on an asymptotically $\mathrm{AdS}_{4}$ background. In the large $N$ limit, the action of the $\mathrm{SO}(8)$ gauge field is Gaussian, and is easily shown to possess electromagnetic (EM) self-duality under which the electric and magnetic fields are interchanged. We demonstrate in Section IIID that it is precisely this EM self-duality of the $3+1$ dimensional gauge field which leads to the constraint (1.9) in the SCFT. Thus the SYM theory obeys a self-duality which is not readily detected in $2+1$ dimensions, but becomes explicit in the holographic theory in $3+1$ dimensions. The generalization of the particle-vortex duality of Abelian CFTs in $D=2+1$ to non-Abelian CFTs is facilitated by the holographic extension to the theory on $\mathrm{AdS}_{4}$.

There have been a few earlier studies connecting dualities in $D=4$ to those in $D=3$. Sethi [56] considered the Kaluza-Klein reduction of S-duality from $D=4$ to $D=3$ by compactifying the $D=4$ theory on a circle in one dimension. This is quite different from the connection above, using a holographic extension. The work of Witten [28] makes a connection which is the same as ours above (see also the work of Leigh and Petkou [57]). He examined the connection between Abelian particle-vortex duality ('mirror symmetry') of CFTs in $D=2+1$ to the action of $\mathrm{SL}(2, Z)$ on Abelian gauge theories on $\mathrm{AdS}_{4}$ at zero temperature. We have considered a similar connection at non-zero temperature for the $\mathcal{N}=8$ SCFT, and shown that it is "holographically self dual" in the large $N$ limit; combined with the non-Abelian $\mathrm{SO}(8)$ symmetry (which implies a single $K$ ), the constraints for the current correlators are stronger than those for Abelian theories.

We will also consider in Appendix E other non-Abelian theories with known gravity descriptions. In particular, we will show that for a theory on a stack of D2 branes, a nontrivial dilaton profile prevents EM self-duality. In this case, we do not have the constraint (1.9), and so find a frequency dependent conductivity.

\section{ABELIAN, NON-COMPACT $\mathbb{C P}^{1}$ MODEL}

This section will consider duality properties and current correlations of the Abelian, easyplane $\mathbb{C P}^{1}$ model of Ref. [16]. This is a theory of two complex scalars $z_{1,2}$ and a non-compact $\mathrm{U}(1)$ gauge field $A_{\mu}$; the non-compactness is necessary to suppress instantons (monopoles), and we indicate below Eq. (2.13) the modifications required when monopoles are present.

More generally, one can consider dualities of the non-compact $\mathbb{C P}^{N-1}$ model where the global $\mathrm{SU}(N)$ flavor symmetry has been explicitly broken down to $\mathrm{U}(1)^{N-1} \times G_{N}$, with $G_{N}$ some subgroup of the permutation group of $N$ objects [42]. The $N=1$ case, which is better known as the Abelian Higgs model, will be described in Appendix B. The $N=2$ case (with $G_{2}=Z_{2}$ ) is described below. The $T>0$ results below have a generalization to all $N>2$, with the mappings spelled out in Ref. [42]. Only the $N=2$ case is self-dual, and this is our 
reason for focusing on it.

It is interesting to note that the duality properties of the non-compact $\mathbb{C} \mathbb{P}^{N-1}$ models have strikingly similar counterparts in $D=2+1$ theories with $\mathcal{N}=4$ supersymmetry [39-41]. In particular, the correspondence is to the theories with one $\mathrm{U}(1)$ vector (gauge) multiplet and $N$ matter hypermultiplets (SQED- $N$ ). SQED-1 is dual to a theory of a single hypermultiplet, with no vector multiplet ${ }^{5}$; this corresponds to the duality, reviewed in Appendix $\mathrm{B}$, of the Abelian Higgs model to the theory of a single complex scalar with no gauge field (also known as the XY model or the $\mathrm{O}(2) \varphi^{4}$ field theory). Next, SQED-2 is self-dual, as is our $N=2$ case. For $N>2$, the dual of SQED- $N$ is a quiver gauge theory, as is the case for the $\mathbb{C P}^{N-1}$ models [42]. ${ }^{6}$ Our results below for $T>0$ should have straightforward extensions to these $\mathcal{N}=4$ supersymmetric theories.

\section{A. Conserved currents}

Let us now begin our analysis of the non-supersymmetric $N=2$ case. The action of the non-compact $\mathbb{C P}^{1}$ theory is

$$
\begin{gathered}
\mathcal{S}=\int d^{2} x d t\left[\left|\left(\partial_{\mu}-i A_{\mu}\right) z_{1}\right|^{2}+\left|\left(\partial_{\mu}-i A_{\mu}\right) z_{2}\right|^{2}+s\left(\left|z_{1}\right|^{2}+\left|z_{2}\right|^{2}\right)+u\left(\left|z_{1}\right|^{2}+\left|z_{2}\right|^{2}\right)^{2}\right. \\
\left.+v\left|z_{1}\right|^{2}\left|z_{2}\right|^{2}+\frac{1}{2 e^{2}}\left(\epsilon^{\mu \nu \lambda} \partial_{\nu} A_{\lambda}\right)^{2}\right]
\end{gathered}
$$

with $u>0$ and $-4 u<v<0$. For these negative values of $v$, the phase for $s$ sufficiently negative has $\left|\left\langle z_{1}\right\rangle\right|=\left|\left\langle z_{2}\right\rangle\right| \neq 0$. We can also define a gauge-invariant vector order parameter $\vec{N}=z^{*} \vec{\sigma} z$, where $\vec{\sigma}$ are the Pauli matrices, and the constraint $v<0$ implies that $\vec{N}$ prefers to lie in the $x y$ plane: hence 'easy-plane' (for $v>0, \vec{N}$ would be oriented along the $z$ 'easy-axis', realizing an Ising order parameter). The $\mathbb{C P}^{1}$ model is usually defined with fixed length constraint $\left|z_{1}\right|^{2}+\left|z_{2}\right|^{2}=1$, but here we have only implemented a soft constraint by the quartic term proportional to $u$; we expect that the models with soft and hard constraints have the same critical properties. We are interested in the nature of the quantum phase transition accessed by tuning the value of $s$ to a critical value $s=s_{c}$. For $s>s_{c}$, we have a 'Coulomb' phase $\langle\vec{N}\rangle=0$ with a gapless photon, while for $s<s_{c}$ there is a 'Higgs' phase with $\langle\vec{N}\rangle \neq 0$. The phase diagram [16] of the model in the $s, T$ plane is shown in Fig. 1 . Both the Higgs and Coulomb phases have phase transitions as the temperature is raised: for the former it is driven by the loss of the Higgs (quasi)-long-range order, while for the latter it

\footnotetext{
${ }^{5}$ The theory of a single hypermultiplet is free. This is because the Gaussian fixed point is protected by $\mathcal{N}=4$ supersymmetry [41]. In the non-supersymmetric case, the Gaussian fixed point is unstable to the interacting Wilson- Fisher fixed point.

${ }^{6}$ A quiver gauge theory consists of a direct product of gauge group factors along with matter fields transforming in the bifundamental representation of pairs of group factors. The word quiver is used because the bifundamental fields are often represented as arrows.
} 


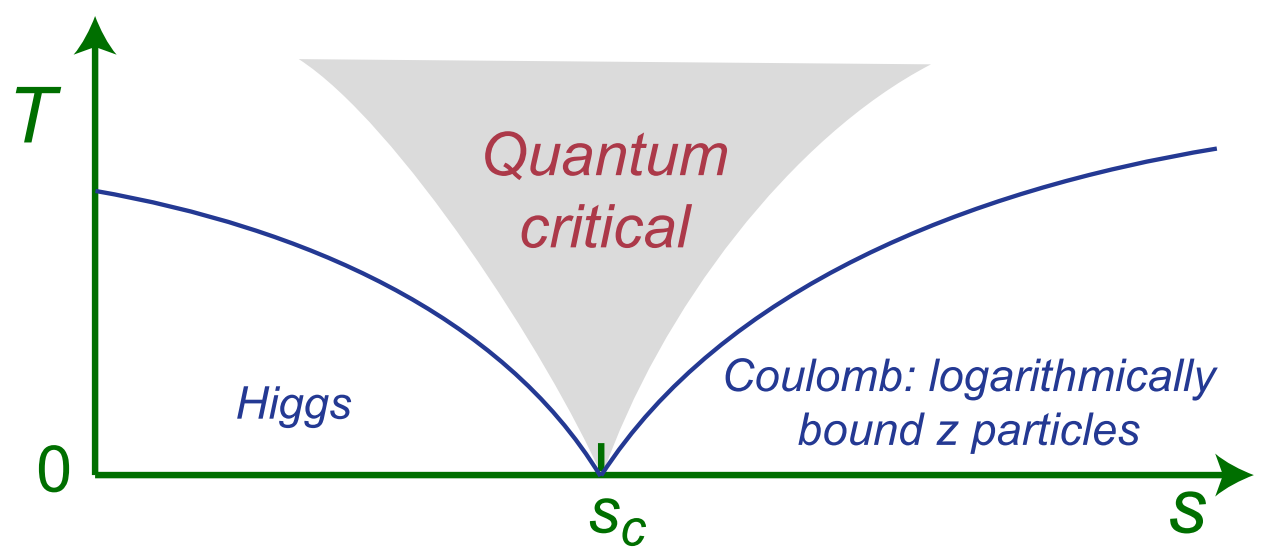

FIG. 1: Phase diagram [16] of the easy-plane non-compact $\mathbb{C P}^{1}$ model (Eq. (2.1)) in 2 spatial dimensions as a function of the coupling $s$ and temperature $T$. The quantum critical point is at $s=s_{c}, T=0$. The finite $T$ correlations of the CFT describe the shaded quantum critical region; the boundary of the shaded region is a crossover into a different physical region, not a phase transition. The full lines are Kosterlitz-Thouless (KT) phase transitions. The KT line for $s<s_{c}$ describes the disappearance of quasi-long-range $x y$ order of $\vec{N}$. The KT transition for $s>s_{c}$ describes the deconfinement of $z$ quanta which are logarithmically bound by the Coulomb interaction in the low temperature phase into particle-anti-particle pairs. The phase diagram can also be described in terms of the dual $w$ theory in Eq. (2.11). Duality interchanges the two sides of $s=s_{c}$ ( $T$ remains invariant under duality), and the $z$ Coulomb phase is interpreted as a $w$ Higgs phase and vice versa.

is a "confinement-deconfinement" transition of the $z$ particle-anti-particle pairs formed from the logarithmic Coulomb force. Neither of these transitions is of interest to us in this paper. Rather, we will compute $T>0$ correlations of the CFT associated with the quantum critical point, and these describe the physical properties of the shaded quantum critical region in Fig. 1.

The theory has a discrete $Z_{2}$ symmetry which exchanges $z_{1}$ and $z_{2}$. The continuous symmetries are a gauge $\mathrm{U}(1)$ symmetry

$$
z_{1} \rightarrow z_{1} e^{i \phi} \quad ; \quad z_{2} \rightarrow z_{2} e^{i \phi} \quad ; \quad A_{\mu} \rightarrow A_{\mu}+\partial_{\mu} \phi
$$

and a global $\mathrm{U}(1)$ symmetry

$$
z_{1} \rightarrow z_{1} e^{i \varphi} ; z_{2} \rightarrow z_{2} e^{-i \varphi}
$$

Associated with these symmetries we can define two currents

$$
J_{1 \mu}=i\left(z_{1}^{*}\left(\partial_{\mu}-i A_{\mu}\right) z_{1}-z_{1}\left(\partial_{\mu}+i A_{\mu}\right) z_{1}^{*}\right)+i\left(z_{2}^{*}\left(\partial_{\mu}-i A_{\mu}\right) z_{2}-z_{2}\left(\partial_{\mu}+i A_{\mu}\right) z_{2}^{*}\right) .
$$

and

$$
J_{2 \mu}=i\left(z_{1}^{*} \partial_{\mu} z_{1}-z_{1} \partial_{\mu} z_{1}^{*}\right)-i\left(z_{2}^{*} \partial_{\mu} z_{2}-z_{2} \partial_{\mu} z_{2}^{*}\right)
$$


Note that $J_{1}$ is even under the $Z_{2}$ symmetry, while $J_{2}$ is odd. Current conservation implies that at $T>0$ these have two-point correlators of the form in Eq. (1.4), with the 4 distinct functions $K_{1,2}^{L, T}$.

Now consider the correlators of the gauge field $A_{\mu}$. It is useful to write this in terms of the leading quadratic terms in the Coleman-Weinberg effective potential:

$$
\begin{aligned}
W=\frac{1}{2} \int_{k, \omega}\left\{-\left(k_{i} A_{0}+\omega A_{i}\right)^{2}\left[\frac{1}{e^{2}}+\frac{\Pi^{L}(k, \omega)}{-\omega^{2}+k^{2}}\right]\right. \\
\left.\quad+A_{i} A_{j}\left(\delta_{i j}-\frac{k_{i} k_{j}}{k^{2}}\right)\left[\frac{k^{2}}{e^{2}}+\Pi^{T}(k, \omega)+\frac{\Pi^{L}(k, \omega) \omega^{2}}{-\omega^{2}+k^{2}}\right]\right\}+\ldots
\end{aligned}
$$

where $\Pi^{L, T}$ are the two components of the photon self energy (the 'polarization' operator); these are related to the current correlations by $\Pi^{L, T}=\sqrt{p^{2}} K_{1}^{L, T}$.

A key point is that at the conformal fixed point describing the phase transition at the quantum critical point $s=s_{c}$ we can safely take the limit $e \rightarrow \infty$ in the above. This is because $\operatorname{dim}[\Pi]=1$, and so the induced polarizations are more singular than the bare Maxwell term. This is a very generic property of CFTs with gauge fields in $D=2+1$. From the effective potential we can obtain the form of the gauge-invariant two-point correlators in the critical regime (it is easiest to work this out in the Coulomb gauge $k_{i} A_{i}=0$ ):

$$
\begin{aligned}
\left\langle\epsilon_{i j} k_{i} A_{j} ; \epsilon_{i^{\prime} j^{\prime}} k_{i^{\prime}} A_{j^{\prime}}\right\rangle & =\frac{k^{2}}{\Pi^{T}(k, \omega)}, \\
\left\langle\epsilon_{i^{\prime} j^{\prime}} k_{i^{\prime}} A_{j^{\prime}} ;\left(k_{i} A_{0}+\omega A_{i}\right)\right\rangle & =\epsilon_{i^{\prime} i} \frac{\omega k_{i^{\prime}}}{\Pi^{T}(k, \omega)}, \\
\left\langle\left(k_{i} A_{0}+\omega A_{i}\right) ;\left(k_{j} A_{0}+\omega A_{j}\right)\right\rangle & =\left(\delta_{i j}-\frac{k_{i} k_{j}}{k^{2}}\right) \frac{\omega^{2}}{\Pi^{T}(k, \omega)}-\frac{k_{i} k_{j}}{k^{2}} \frac{\left(-\omega^{2}+k^{2}\right)}{\Pi^{L}(k, \omega)} .
\end{aligned}
$$

\section{B. Vortices and duality}

Here we will build a dual description of the $\mathbb{C P}^{1}$ model, treating the vortices of the original model as complex scalar fields in the dual description. Consider the topological vortex excitations in the Higgs state of the action (2.1). These are characterized [58] by a pair of winding numbers $\left(n_{1}, n_{2}\right)$ associated with the phases of $z_{1}$ and $z_{2}$ out at spatial infinity. In general, such a vortex has a logarithmically diverging energy because the currents are only partially screened by the gauge field $A_{\mu}$. By an extension of the Abrikosov-Nielsen-

Olesen argument, it can be seen that the co-efficient of the logarithmically divergent energy is proportional to

$$
\left(2 \pi n_{1}-\int d^{2} x \epsilon_{i j} \partial_{i} A_{j}\right)^{2}+\left(2 \pi n_{2}-\int d^{2} x \epsilon_{i j} \partial_{i} A_{j}\right)^{2},
$$

and this is minimized when the total $A_{\mu}$ flux is quantized as $[16,42,58]$

$$
\int d^{2} x \epsilon_{i j} \partial_{i} A_{j}=\pi\left(n_{1}+n_{2}\right) .
$$


Let us now identify the $(1,0)$ vortex as the worldline of a dual particle $w_{1}$, the $(0,1)$ vortex as the worldline of a dual particle $w_{2}$, and try to construct a dual theory by introducing complex scalar fields $w_{1}(x), w_{2}(x)$. Then from Eq. (2.9), Lorentz covariance implies that the total $w$ current is related to the $A_{\mu}$ flux:

$$
\frac{1}{\pi} \epsilon_{\mu \nu \lambda} \partial^{\nu} A^{\lambda}=i\left(w_{1}^{*} \partial_{\mu} w_{1}-w_{1} \partial_{\mu} w_{1}^{*}\right)+i\left(w_{2}^{*} \partial_{\mu} w_{2}-w_{2} \partial_{\mu} w_{2}^{*}\right) .
$$

A second key property is that there are forces with a logarithmic potential between the $w_{1,2}$ particles. These are also easily seen from the structure of the classical vortex solutions of Eq. (2.1). Also, it is the difference of the $z_{1}$ and $z_{2}$ currents, which is not screened by the $A_{\mu}$ field, which contributes to an attractive logarithmic potential between the $w_{1}$ and $w_{2}$ particles. Another way to see this is to consider the configuration of the gaugeinvariant Higgs field $\left(N_{x}, N_{y}\right)$ around each vortex: the $w_{1}$ has an anti-clockwise winding of the $\arg \left(N_{x}+i N_{y}\right)$, while the $w_{2}$ has a clockwise winding. Because there is a finite stiffness associated with this Higgs order, a $w_{1}$ particle will attract a $w_{2}$ particle, while two $w_{1}$ (or $w_{2}$ ) particles will repel each other.

We can now guess the form of the effective theory for the $w_{1,2}$ particles. We mediate that logarithmic potential as the Coulomb potential due to a new 'dual' gauge field $\widetilde{A}_{\mu}$. Then general symmetry arguments and the constraints above imply the dual theory [16]

$$
\begin{gathered}
\widetilde{\mathcal{S}}=\int d^{2} x d t\left[\left|\left(\partial_{\mu}-i \widetilde{A}_{\mu}\right) w_{1}\right|^{2}+\left|\left(\partial_{\mu}+i \widetilde{A}_{\mu}\right) w_{2}\right|^{2}+\widetilde{s}\left(\left|w_{1}\right|^{2}+\left|w_{2}\right|^{2}\right)+\widetilde{u}\left(\left|w_{1}\right|^{2}+\left|w_{2}\right|^{2}\right)^{2}\right. \\
\left.+\widetilde{v}\left|w_{1}\right|^{2}\left|w_{2}\right|^{2}+\frac{1}{2 \widetilde{e}^{2}}\left(\epsilon^{\mu \nu \lambda} \partial_{\nu} \widetilde{A}_{\lambda}\right)^{2}\right]
\end{gathered}
$$

Note especially the difference in the charge assignments from (2.1) - now the $w_{1,2}$ particles have opposite charges under $\widetilde{A}_{\mu}$. Apart from this, the theories have an identical form, and so current correlation functions $\widetilde{K}_{1,2}^{L, T}$, associated with the global and gauge U(1) symmetries, will have the same dependence upon the couplings in $\widetilde{\mathcal{S}}$ as the $K_{1,2}^{L, T}$ have on $\mathcal{S}$. However, the explicit expressions for the current in terms of the field operators have a sign interchanged:

$$
\widetilde{J}_{1 \mu}=i\left(w_{1}^{*}\left(\partial_{\mu}-i \widetilde{A}_{\mu}\right) w_{1}-w_{1}\left(\partial_{\mu}+i \widetilde{A}_{\mu}\right) w_{1}^{*}\right)-i\left(w_{2}^{*}\left(\partial_{\mu}+i \widetilde{A}_{\mu}\right) w_{2}-w_{2}\left(\partial_{\mu}-i \widetilde{A}_{\mu}\right) w_{2}^{*}\right)
$$

and

$$
\widetilde{J}_{2 \mu}=i\left(w_{1}^{*} \partial_{\mu} w_{1}-w_{1} \partial_{\mu} w_{1}^{*}\right)+i\left(w_{2}^{*} \partial_{\mu} w_{2}-w_{2} \partial_{\mu} w_{2}^{*}\right) .
$$

We note in passing the extension of the above analysis to a compact $\mathbb{C P}^{1}$ theory of the $z$ particles. Following Polyakov [59], we have to include monopoles which change the $A_{\mu}$ flux by $2 \pi$. This can be achieved by adding the term $-y_{m}\left(w_{1} w_{2}+w_{1}^{*} w_{2}^{*}\right)$ to the $w$ action $\widetilde{\mathcal{S}}$, where $y_{m}$ is the monopole fugacity. This monopole operator is neutral under $\widetilde{A}_{\mu}$ charge and, from Eq. (2.10), catalyzes the required change in $A_{\mu}$ flux. This is a relevant perturbation: the theories for the $z$ and $w$ particles are no longer equivalent under duality, and the universality 
class of the transition is changed. We will not consider the compact case further; for more details, see the review [60].

Returning to the non-compact theory, we note the duality mapping can now also be carried backwards from the $w$ theory to the $z$ theory, and from (2.10) we see that the theories $\mathcal{S}$ and $\widetilde{\mathcal{S}}$ are connected by the relations

$$
\begin{aligned}
& \frac{1}{\pi} \epsilon_{\mu \nu \lambda} \partial^{\nu} A^{\lambda}=\widetilde{J}_{2 \mu}, \\
& \frac{1}{\pi} \epsilon_{\mu \nu \lambda} \partial^{\nu} \widetilde{A}^{\lambda}=J_{2 \mu} .
\end{aligned}
$$

From these relations, Eq. (2.7), and the definition (1.4), we immediately obtain the relation between $K_{1}$ and $K_{2}$ :

$$
\begin{array}{ll}
K_{1}^{T}(\omega, k) \widetilde{K}_{2}^{L}(\omega, k)=\frac{1}{\pi^{2}}, & \widetilde{K}_{1}^{T}(\omega, k) K_{2}^{L}(\omega, k)=\frac{1}{\pi^{2}} \\
K_{1}^{L}(\omega, k) \widetilde{K}_{2}^{T}(\omega, k)=\frac{1}{\pi^{2}}, & \widetilde{K}_{1}^{L}(\omega, k) K_{2}^{T}(\omega, k)=\frac{1}{\pi^{2}} .
\end{array}
$$

Now, assuming a single second-order transition obtained by tuning the parameter $s$, the above reasoning implies that this critical point must be self-dual, $K_{1}^{T, L}=\widetilde{K}_{1}^{T, L}$, and $K_{2}^{T, L}=$ $\widetilde{K}_{2}^{T, L}$. Self-duality thus immediately implies relation (1.8), as claimed in the Introduction.

Monte Carlo simulations [61] of a current loop model related to $\mathcal{S}$ observe a weak firstorder transition. This is possibly because they are using a particular lattice action which is not within the domain of attraction of the self-dual point. In any case, the duality mappings between the two phases on either side of the transition apply, and the constraints on a possible CFT remain instructive.

\section{THE M2-BRANE THEORY}

This section examines the transport properties of the non-Abelian $\mathrm{SU}(N)$ Yang Mills theory in $D=2+1$ with $\mathcal{N}=8$ supersymmetry. The weak- coupling action and field content of this theory is most directly understood by dimensional reduction of the $\mathcal{N}=1$ SYM theory in $D=9+1$ on the flat torus $T^{7}$ [62]. This reduction shows that the $D=2+1$ theory has an explicit $\mathrm{SO}(7) \mathrm{R}$-charge global symmetry. The $D=9+1 \mathrm{SYM}$ theory has only a single gauge coupling constant, and therefore, so does the $D=2+1$ theory. The latter coupling has a positive scaling dimension, and flows to strong-coupling in the infrared. It is believed [43] that the flow is to an infrared-stable fixed point that describes a SCFT. It was also argued that this SCFT has an emergent R-charge symmetry which is expanded to $\mathrm{SO}(8)$. We shall be interested in the transport properties of this $\mathrm{SO}(8) \mathrm{R}$-charge in the $\mathrm{SCFT}$ at $T>0$ in the present section.

We are faced by a strongly-coupled SCFT, and a perturbative analysis of the field theory described above is not very useful. Instead, remarkable progress is possible using the 
connection to string theory and the AdS/CFT correspondence. The $D=2+1$ SYM theory is contained in the low energy description of Type IIA string theory in the presence of a stack of $N$ D2-branes. The flow to strong coupling of the SYM theory corresponds in string theory to the lift of ten-dimensional Type IIA strings to eleven-dimensional M-theory [46]. So we can directly access the $D=2+1$ SYM SCFT by considering M-theory in the presence of a stack of $N$ M2-branes [45]. In the large $N$ limit, M-theory can be described by the semiclassical theory of eleven-dimensional supergravity, and this will be our main tool in the analysis described below. This formulation also makes the $\mathrm{SO}(8)$ R-charge symmetry explicit, because the M2-branes curve the spacetime of eleven-dimensional supergravity to $\mathrm{AdS}_{4} \times S^{7}$.

Another powerful feature of the supergravity formulation is that it can be extended to $T>$ 0 . We have to consider supergravity in a spacetime which is asymptotically $\mathrm{AdS}_{4}$, but which also contains a black hole. The Hawking temperature of the black hole then corresponds to the temperature of the SCFT [63] (for example, fluctuation-dissipation theorems are satisfied [48]). Hydrodynamics of the SCFT emerges from the semiclassical supergravity dynamics in the presence of the black hole. ${ }^{7}$

Turning to our explicit computation of dynamics in M-theory, we consider the gravitational background associated with a stack of $N$ M2-branes, with $N \gg 1$ [45, 49, 65],

$$
d s^{2}=\frac{r^{4}}{R^{4}}\left[-f(r) d t^{2}+d x^{2}+d y^{2}\right]+\frac{R^{2}}{r^{2}}\left[\frac{d r^{2}}{f(r)}+r^{2} d \Omega_{7}^{2}\right],
$$

where $f(r)=1-r_{0}^{6} / r^{6}$. It is more convenient for us to change coordinates from $r$ to $u=\left(r_{0} / r\right)^{2}$, in terms of which

$$
d s^{2}=\frac{r_{0}^{4}}{R^{4} u^{2}}\left[-f(u) d t^{2}+d x^{2}+d y^{2}\right]+\frac{R^{2}}{4 u^{2} f} d u^{2}+R^{2} d \Omega_{7}^{2}
$$

and $f(u)=1-u^{3}$. The horizon of the black hole is located at $u=1$, and the boundary of $\mathrm{AdS}_{4}$ is at $u=0$.

The relationship between the quantities in the worldvolume SCFT ( $N$ and temperature $T)$ and those of the metric $\left(R\right.$ and $\left.r_{0}\right)$ are given by $[45,49]$

$$
\pi^{5} R^{9}=\sqrt{2} N^{3 / 2} \kappa^{2}, \quad T=\frac{3}{2 \pi} \frac{r_{0}^{2}}{R^{3}},
$$

where $\kappa$ is the gravitational coupling strength of $D=10+1$ supergravity.

There is a precise correspondence between correlation functions computed in the $\mathrm{D}=2+1$ CFT and correlation functions of supergravity fields computed in the metric (3.1) [46-48]. We will use this to compute charge transport properties.

\footnotetext{
${ }^{7}$ Strictly speaking, the appearance of a black hole is dual to being at finite temperature and being in a deconfined phase; it is possible to have a finite temperature gravitational description without a black hole $[63,64]$.
} 
In the metric (3.1) a 7 -sphere factors out: $R^{2} d \Omega_{7}^{2}$. The spacetime thus has a $\mathrm{SO}(8)$ symmetry. This matches with the global symmetry in the M2 worldvolume theory: there is a R-charge which transforms under the same global symmetry. The following subsections will compute the two-point correlations of the R-charge currents, $J_{a \mu}$, with $a=1, \ldots, 28$.

The existence of a compact 7 -sphere makes it possible to do Kaluza-Klein reduction on this space. We expand all fields in terms of spherical harmonics on the 7-sphere. The original fields of M-theory are the metric tensor $g_{\mu \nu}$ and a three-index antisymmetric tensor $A_{\mu \nu \lambda}$. Upon Kaluza-Klein reduction, an $\mathrm{SO}(8)$ gauge field appears from the components of the metric and the three-form where only one index is in the $\operatorname{AdS}_{4}$ directions $(t, x, y$, and $u$ ) and the others are in the $S^{7}$ directions (see Appendix $\mathrm{C}$ for details). The action for this gauge field is

$$
S=-\frac{1}{4 g_{4 \mathrm{D}}^{2}} \int d^{4} x \sqrt{-g} g^{M A} g^{N B} F_{M N}^{a} F_{A B}^{a},
$$

where uppercase Latin indices $A, B, M, N$ run four values of $t, x, y$, and $u$ (in contrast to Greek indices $\alpha, \beta, \mu, \nu$ which run $t, x$ and $y)$. The four-dimensional gauge coupling constant $g_{4 \mathrm{D}}$ is dimensionless, and its large $N$ value is computed in Appendix $\mathrm{C}$

$$
\frac{1}{g_{4 \mathrm{D}}^{2}}=\frac{\sqrt{2}}{6 \pi} N^{3 / 2} .
$$

Although we focus on the gravity background constructed from a stack of $N$ M2-branes in flat 11-dimensional space, there are a number of related examples which are easily understood from considering (3.4). The key observation, which we discuss further in Section IIID, is that (3.4) exhibits classical electric-magnetic duality. In the case of our M2-brane theory, this duality is close enough to a self-duality to enforce a relation on the current-current two point functions and result in a frequency independent conductivity. In fact, this self-duality holds in a more general context. Consider an eleven dimensional space which factorizes into $\mathbb{R}^{2,1}$ and a Calabi-Yau four-fold which develops a local singularity. By placing a stack of M2-branes at the singularity, we should obtain a more exotic $2+1$ dimensional conformal field theory which still has at least a U(1) global R-symmetry. Kaluza-Klein reduction of the gravity theory will yield precisely (3.4) and our results on holographic self-duality will carry over to these more general cases.

There are two other interesting generalizations to consider in which holographic selfduality fails. After Kaluza-Klein reduction, the gauge fields $F_{A B}$ will support electrically charged black holes [66]. These black holes are dual to introducing an R-charge chemical potential to the field theory. Another interesting $2+1$ dimensional field theory with a holographic description is the theory living on a stack of D2-branes in type IIA string theory. In both cases, there is generically a nontrivial scalar which appears in a modification of (3.4) as a coupling constant which depends on the holographic radial direction. The relation on the two-point functions will be between a theory with coupling $g_{4 \mathrm{D}}(u)$ and one with coupling $1 / g_{4 \mathrm{D}}(u)$. For details concerning this more general perspective, see Appendix E. 


\section{A. Current-current correlators}

We now proceed to the computation of the two-point correlators of the $J_{a \mu}$ in the CFT at $T>0$. Here we will work in Minkowski space (real frequencies and time), and so define the current correlation as follows:

$$
C_{\mu \nu}(x-y) \delta_{a b}=-i \theta\left(x^{0}-y^{0}\right)\left\langle\left[J_{a \mu}(x), J_{a \nu}(y)\right]\right\rangle
$$

The $\delta_{a b}$ follows from $\mathrm{SO}(8)$ symmetry. The expectation value is taken in a translationinvariant state, so we can Fourier transform to $C_{\mu \nu}(p)$, where $p_{\mu}=(-\omega, \boldsymbol{k})$. Spectral density is proportional to the imaginary part of the retarded function,

$$
\rho_{\mu \nu}(p)=-2 \operatorname{Im} C_{\mu \nu}(p)
$$

It is an odd, real function of $p$, whose diagonal components are positive (for positive frequency). Expectation values of all global conserved charges are assumed to vanish in the equilibrium state; in other words we consider systems without chemical potentials. Conservation of $J_{a \mu}(x)$ implies that the correlation functions may be defined so that they satisfy the Ward identity ${ }^{8} p^{\mu} C_{\mu \nu}(p)=0$. Then, as in Section I and in Eq. (1.4), we can write $C_{\mu \nu}$ in the form

$$
C_{\mu \nu}(p)=P_{\mu \nu}^{T} \Pi^{T}(\omega, k)+P_{\mu \nu}^{L} \Pi^{L}(\omega, k) .
$$

(The relationship between $\Pi$ and $K$ is $\Pi^{T, L}=\sqrt{p^{2}} K^{T, L}$.) Without loss of generality one can take the spatial momentum oriented along the $x$ direction, so that $p=(\omega, k, 0)$. Then the components of the retarded current-current correlation function are

$$
C_{y y}(\omega, k)=\Pi^{T}(\omega, k)
$$

as well as

$$
C_{t t}=\frac{k^{2}}{\omega^{2}-k^{2}} \Pi^{L}(\omega, k), \quad C_{t x}=C_{x t}=\frac{-\omega k}{\omega^{2}-k^{2}} \Pi^{L}(\omega, k), \quad C_{x x}=\frac{\omega^{2}}{\omega^{2}-k^{2}} \Pi^{L}(\omega, k) .
$$

\section{B. Correlation functions from AdS/CFT}

In order to find the retarded function, one needs to study fluctuations of vector fields on the background spacetime created by a stack of M2-branes. At the linear order the fields satisfy the equations

$$
\partial_{M}\left(\sqrt{-g} g^{M A} g^{N B} F_{A B}\right)=0
$$

\footnotetext{
8 One may choose to define the correlation functions in such a way that local (in position space) counterterms appear on the right-hand side of the Ward identities. The correlation functions defined in this way will differ from $C_{\mu \nu}(p)$ by analytic functions of $\omega$ and $\boldsymbol{k}$.
} 
These equations are to be solved with the boundary conditions

$$
\lim _{u \rightarrow 0} A_{\mu}(u, x)=A_{\mu}^{0}(x),
$$

at $u=0$. Near $u=1$ one imposes the outgoing-wave boundary condition, which means that for $u$ slightly less than 1 the solution is purely a wave that propagates toward the horizon. Due to translational invariance with respect to $x$ one can solve for each Fourier mode $e^{i p \cdot x}$ separately. The result can be represented in the form

$$
A_{\mu}(u, p)=M_{\mu}^{\nu}(u, p) A_{\nu}^{0}(p) .
$$

Then, according to the AdS/CFT prescription formulated in Ref. [47], the current-current correlator can be found from the formula ${ }^{9}$

$$
C_{\mu \nu}(p)=-\chi \lim _{u \rightarrow 0} M_{\mu \nu}^{\prime}(u, p),
$$

where $\chi$ is the constant that appears in the normalization of the action,

$$
S=\frac{\chi}{2} \int d u d^{3} x\left(A_{t}^{\prime 2}-f A_{x}^{\prime 2}-f A_{y}^{\prime 2}+\ldots\right)
$$

(only terms with two derivatives with respect to $u$ are written). In our case $\chi=4 \pi T / 3 g_{4 \mathrm{D}}^{2}$. It turns out that $\chi$ is precisely the charge susceptibility. ${ }^{10}$

The prescription given above might appear ad-hoc. However it is a special case of a more general AdS/CFT prescription that gives real-time correlators of any number of operators [48]. For our task, however, the above prescription is technically most straightforward to implement.

We work in the radial gauge $A_{u}=0$, and take all fields $A_{\mu}(x)$ to be proportional to $e^{-i \omega t+i \boldsymbol{k} \cdot \boldsymbol{x}}$. Taking momentum $\boldsymbol{k}$ along the $x$ direction, $\boldsymbol{k}=(k, 0)$, one finds that the fluctuating vector fields satisfy the following equations [49]

$$
\begin{aligned}
w A_{t}^{\prime}+q f A_{x}^{\prime} & =0, \\
A_{t}^{\prime \prime}-\frac{1}{f}\left(w q A_{x}+q^{2} A_{t}\right) & =0, \\
A_{x}^{\prime \prime}+\frac{f^{\prime}}{f} A_{x}^{\prime}+\frac{1}{f^{2}}\left(w q A_{t}+w^{2} A_{x}\right) & =0, \\
A_{y}^{\prime \prime}+\frac{f^{\prime}}{f} A_{y}^{\prime}+\frac{1}{f^{2}}\left(w^{2}-q^{2} f\right) A_{y} & =0 .
\end{aligned}
$$

Here prime denotes derivative with respect to $u$; $w$ and $q$ are the dimensionless frequency and momentum, $w \equiv 3 \omega /(4 \pi T), q \equiv 3 k /(4 \pi T)$. Note that the equation for the transverse

${ }^{9}$ Greek indices on $M_{\mu \nu}$ are raised using the flat space Minkowski metric.

10 The hydrodynamic density-density response function found in [49] is $C_{t t}=\left(1 / g_{4 \mathrm{D}}^{2}\right) k^{2} /\left(i \omega-D_{c} k^{2}\right)$. Comparing this to the hydrodynamic form $C_{t t}=\chi D_{c} k^{2} /\left(i \omega-D_{c} k^{2}\right)$, we find the above value for charge susceptibility $\chi$. 
potential $A_{y}$ decouples from the rest. Moreover, Eq. (3.18) can be shown to follow from Eqs. (3.16) and (3.17) and so is not independent. Combining Eqs. (3.16) and (3.17) one can obtain an equation that does not involve $A_{x}$,

$$
A_{t}^{\prime \prime \prime}+\frac{f^{\prime}}{f} A_{t}^{\prime \prime}+\frac{1}{f^{2}}\left(w^{2}-q^{2} f\right) A_{t}^{\prime}=0 .
$$

One can think about this equation as a second-order equation for $A_{t}^{\prime}$. It was observed in [49] that Eq. (3.20) has the same form as the equation for $A_{y}$. Such degeneracy is unusual, and we now proceed to explore its implications.

\section{Transverse channel}

Let us start with the retarded function for transverse currents, $C_{y y}(\omega, \boldsymbol{k})$. According to the AdS/CFT prescription (3.14),

$$
C_{y y}(p)=-\chi \lim _{u \rightarrow 0} M_{y y}^{\prime}(u, p)
$$

The function $M_{y y}(u, p)$ is the solution to Eq. (3.19) which satisfies the outgoing-wave boundary condition on the horizon $u=1$, and $M_{y y}(0, p)=1$ at the boundary $u=0$.

Let us denote a solution to Eq. (3.19) which satisfies the outgoing boundary condition at the horizon as $\psi(u)$. The normalization of $\psi(u)$ is left arbitrary. Near $u=0$, Eq. (3.19) allows two asymptotic solutions, which can be expressed in terms of the Frobenius series,

$$
\begin{aligned}
Z_{I}(u) & =1+h Z_{I I}(u) \ln u+b_{I}^{(1)} u+\ldots, \\
Z_{I I}(u) & =u\left(1+b_{I I}^{(1)} u+b_{I I}^{(2)} u^{2}+\ldots\right) .
\end{aligned}
$$

The coefficient $b_{I}^{(1)}$ is arbitrary, and we set it to zero. All other coefficients are determined by substituting expansion (3.25) in the original equation (3.19). In particular, we find that $h=0$, therefore

$$
\begin{aligned}
Z_{I}(0)=1, & Z_{I}^{\prime}(0)=0 \\
Z_{I I}(0)=0, & Z_{I I}^{\prime}(0)=1 .
\end{aligned}
$$

The outgoing-wave solution $\psi(u)$ can be exprressed as

$$
\psi(u)=\mathcal{A} Z_{I}(u)+\mathcal{B} Z_{I I}(u),
$$

where $\mathcal{A}$ and $\mathcal{B}$ depend on the parameters of the equation, in particular on $w$ and $q$. From Eq. (3.24) it follows that $\psi(0)=\mathcal{A}$ and $\psi^{\prime}(0)=\mathcal{B}$. The properly normalized mode function is $M_{y y}(u, p)=\psi(u) / \psi(0)$, and therefore we find

$$
C_{y y}(w, q)=-\chi \frac{\mathcal{B}(w, q)}{\mathcal{A}(w, q)} .
$$




\section{Longitudinal channel}

Let us now look at the correlators in the longitudinal channel: $C_{t t}, C_{t x}$, and $C_{x x}$. For that we need to solve Eqs. (3.16) and (3.17). First, we know that $A_{t}^{\prime}(u)$ satisfies the same equation as $A_{y}(u)$. Therefore, we can write $A_{t}^{\prime}(u)=c \psi(u)$, where $c$ is some coefficient. This coefficient can be fixed from the boundary conditions at $u=0$ by employing Eqs. $(3.17)$ and $\psi^{\prime}(0)=\mathcal{B}$. We find

$$
A_{t}^{\prime}(u)=\left[\frac{\mathcal{A}}{\mathcal{B}} Z_{I}(u)+Z_{I I}(u)\right]\left(w q A_{x}^{0}+q^{2} A_{t}^{0}\right) .
$$

From Eq. (3.16) we also find

$$
A_{x}^{\prime}(u)=-\frac{1}{f}\left[\frac{\mathcal{A}}{\mathcal{B}} Z_{I}(u)+Z_{I I}(u)\right]\left(w^{2} A_{x}^{0}+w q A_{t}^{0}\right) .
$$

These equations are to be compared with Eq. (3.13), from which one extracts $M_{\mu \nu}^{\prime}(u, p)$. Putting $u=0$, one find the correlators

$$
C_{t t}(w, q)=\chi q^{2} \frac{\mathcal{A}(w, q)}{\mathcal{B}(w, q)}, \quad C_{x x}(w, q)=\chi w^{2} \frac{\mathcal{A}(w, q)}{\mathcal{B}(w, q)} .
$$

In Appendix D we show that at zero momentum, $q=0$, the mode equation (3.19) can be solved analytically, which allows one to determine $\Pi^{T}(w, 0)=\Pi^{L}(w, 0)$. However, one can determine the conductivity without explicitly solving the mode equation, as we now show.

\section{Conductivity}

We see that both $C_{y y}$ and $C_{x x}$ are expressed in terms of the same connection coefficients $\mathcal{A}$ and $\mathcal{B}$. Eliminating the coefficients, we find

$$
C_{x x}(w, q) C_{y y}(w, q)=-\chi^{2} w^{2}, \quad C_{t t}(w, q) C_{y y}(w, q)=-\chi^{2} q^{2} .
$$

Expressed in terms of the self-energies $\Pi^{T}, \Pi^{L}$ this reads

$$
\Pi^{T}(w, q) \Pi^{L}(w, q)=-\chi^{2}\left(w^{2}-q^{2}\right) .
$$

Note that this relation holds for all $w$ and $q$ : we have not made any small-frequency approximations anywhere. In fact, we did not even have to solve the mode equations! Combining Eqs. (1.4), (3.8), and (3.31), we obtain our main result in Eq. (1.9).

As discussed in Section I, at zero momentum, rotation invariance implies that $\Pi^{T}=\Pi^{L}$, therefore relation (3.31) uniquely determines the self-energy ${ }^{11} \Pi^{T}(\omega, 0)=\Pi^{L}(\omega, 0)=-i \chi w$ for all $w$. The conductivity is given by $\sigma(\omega / T)=i \Pi^{T}(\omega, 0) / \omega$, and we find

$$
\sigma(\omega / T)=\chi \frac{3}{4 \pi T}=\chi D_{c}=\frac{1}{g_{4 \mathrm{D}}^{2}}
$$

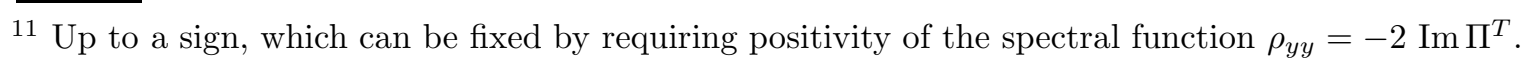


where $D_{c}=3 /(4 \pi T)$ is the diffusion constant found in [49]. Note that the Einstein relation between the conductivity and the diffusion constant is satisfied. Also, as noted earlier, it is surprising that $\sigma(\omega / T)$ is actually independent of $\omega / T$. [Dependence upon $\omega / T$ is found at all non-zero $k$, as is shown below.] This $\omega$-independence is a consequence of the relation (3.31), which in turn follows from the fact that $A_{t}^{\prime}$ and $A_{y}$ satisfy the same equation in the bulk. It can be traced back to the electromagnetic duality of the classical action (3.4), as we now show.

\section{Electric-magnetic duality}

Even though the origin of the relation (3.31) is puzzling from the point of view of the microscopic degrees of freedom in the $\mathcal{N}=8 \mathrm{SCFT}$, its origin from the bulk point of view can be traced to electric-magnetic (EM) duality of an abelian gauge field. Indeed, currentcurrent correlators are computed from the Maxwell equations in the four-dimensional bulk, and it is precisely in four dimensions that Maxwell equations may possess EM duality.

Although in general the R-symmetry may be non-abelian and hence be dual to a nonabelian gauge field in the bulk, we work in the classical supergravity limit and must keep $N$ large. At large $N$, the gauge coupling $g_{4 \mathrm{D}} \propto N^{-3 / 4}$ is very small, and our non-abelian gauge field factorizes into a number of effectively abelian pieces to leading order in $1 / N$.

If we write equations of motion in terms of the gauge-invariant $F_{M N}$ (rather than the vector potential), then Maxwell equations have to be supplemented by a Bianchi identity,

$$
\begin{aligned}
& \partial_{M}\left(\sqrt{-g} F^{M N}\right)=0 \\
& \partial_{M}\left(\sqrt{-g} \frac{1}{2} \varepsilon^{M N A B} F_{A B}\right)=0
\end{aligned}
$$

where $\varepsilon^{M N A B}$ is the totally antisymmetric tensor, with $\varepsilon^{0123}=1 / \sqrt{-g}$. Now, one can introduce $G_{M N}$ defined as $F^{M N}=\frac{1}{2} \varepsilon^{M N A B} G_{A B}$, which can be inverted to give $G^{M N}=$ $-\frac{1}{2} \varepsilon^{M N A B} F_{A B}$. Expressed in terms of $G$, the equations of motion become

$$
\begin{aligned}
& \partial_{M}\left(\sqrt{-g} \frac{1}{2} \varepsilon^{M N A B} G_{A B}\right)=0 \\
& \partial_{M}\left(\sqrt{-g} G^{M N}\right)=0
\end{aligned}
$$

Maxwell equations for $F$ become a Bianchi identity for $G$, and vice versa. $G_{M N}$ is the dual field strength tensor, and we can also define a dual vector potential $B_{M}$ by $G_{M N}=\partial_{M} B_{N}-$ $\partial_{N} B_{M}$. Note that the validity of EM duality does not depend on the background spacetime having any particular symmetries such as Lorentz symmetry, or rotational symmetry.

From the point of view of AdS/CFT, the EM dual theory in the bulk will correspond to some theory on the boundary, which is a dual of the original SCFT. In particular, the dual vector potential $B_{\mu}$ will couple to the dual current $\tilde{J}_{\mu}$, and one can compute two-point functions $C_{\mu \nu}^{\text {dual }}(\omega, k)$ in the dual theory. 

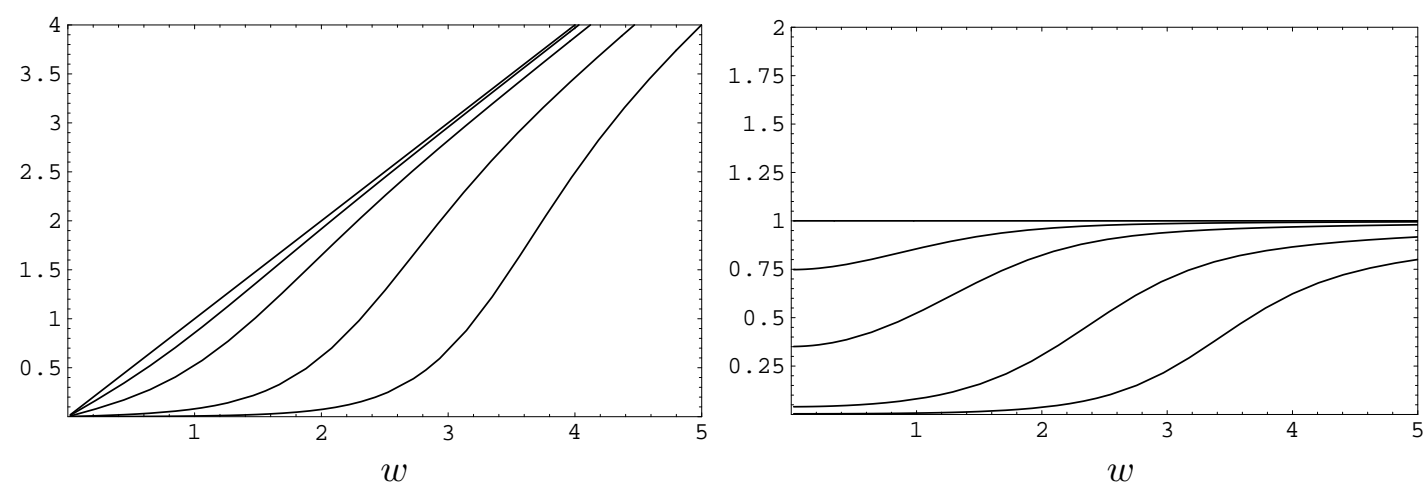

FIG. 2: Imaginary part of the retarded function $C_{y y}(\omega, k)$, plotted in units of $(-\chi)$, as a function of dimensionless frequency $w \equiv 3 \omega /(4 \pi T)$, for several values of dimensionless momentum $q \equiv$ $3 k /(4 \pi T)$. Curves from left to right correspond to $q=0,0.5,1.0,2.0,3.0$. Left: $\operatorname{Im} C_{y y}(w, q)$, Right: $\operatorname{Im} C_{y y}(w, q) / w$.

In components we have $F^{t z}=G_{x y} / \sqrt{-g}$. This means that the equation for $\sqrt{-g} F^{t z}$ obtained from equations (3.33) is the same as the equation for $G_{x y}$, obtained from the dual equations (3.34). In our particular example of the non-extremal M2 background metric, we have $\sqrt{-g} F^{t u} \propto A_{t}^{\prime}(u)$, and $G_{x y} \propto k B_{y}(u)$ (in the radial gauge). Thus the equation for $A_{t}^{\prime}(u)$ is the same as the equation for $B_{y}(u)$. Then, by the argument in section III B we find a relation between the self-energies $\Pi^{T, L}$ in the original theory, and the self-energies $\widetilde{\Pi}^{T, L}$ in the dual theory:

$$
\begin{aligned}
& \Pi^{T}(w, q) \widetilde{\Pi}^{L}(w, q)=-\chi^{2}\left(w^{2}-q^{2}\right) \\
& \widetilde{\Pi}^{T}(w, q) \Pi^{L}(w, q)=-\chi^{2}\left(w^{2}-q^{2}\right)
\end{aligned}
$$

For our M2-branes, EM duality is a self-duality, and the EM dual theory is the same as the original theory, as is evident from equations (3.33), (3.34). Therefore, $C_{\mu \nu}=C_{\mu \nu}^{\text {dual }}$, and $\widetilde{\Pi}^{T}=\Pi^{T}, \widetilde{\Pi}^{L}=\Pi^{L}$. This gives back our main result (3.31). ${ }^{12}$ In the case when there are non-trivial background profiles for scalar fields, the EM dual theory is not equivalent to the original theory. This is discussed in Appendix E.

\section{E. Full spectral functions}

We will now evaluate the spectral functions numerically, for all $\omega$ and $k$. To do so, we find a solution $\psi(u)$ to the mode equation (3.19) with the outgoing boundary conditions at the

12 The present discussion assumes that the coupling constant $g_{4 \mathrm{D}}^{2}$ is not inverted in the dual theory, which is justified for a free, sourceless, abelian gauge field. One could formally repeat the same steps leading to Eq. (3.31), assuming $\widetilde{g}_{4 \mathrm{D}}^{2}=1 / g_{4 \mathrm{D}}^{2}$, as is standard in EM duality. However, in this case the coupling constant $\widetilde{g}_{4 \mathrm{D}}^{2} \propto N^{3 / 2}$ becomes large, invalidating the bulk description in terms of a classical gauge field. 

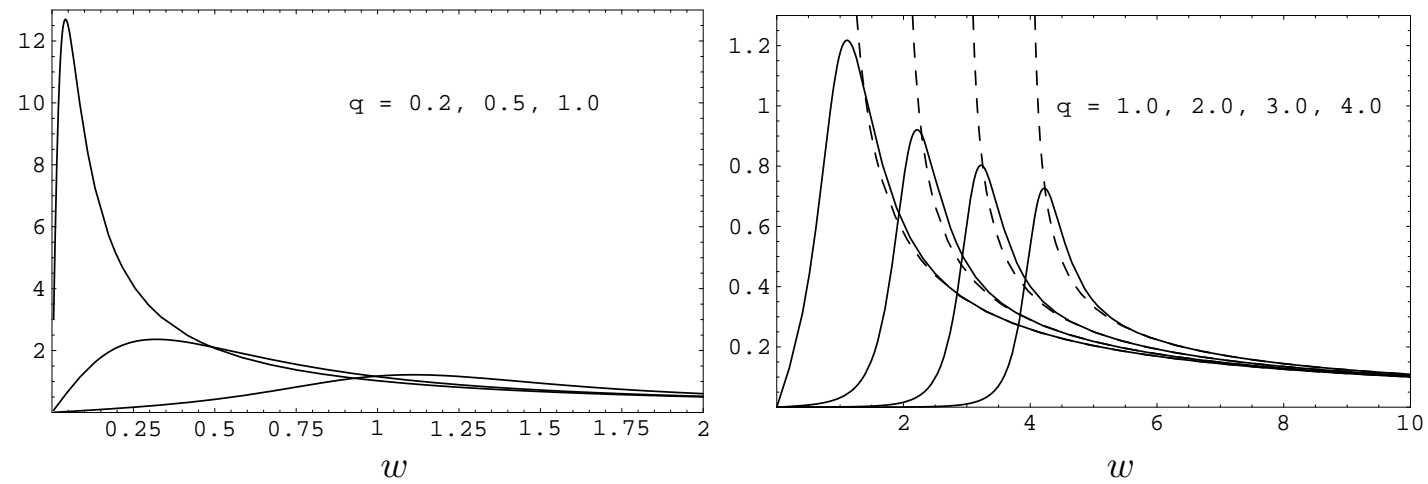

FIG. 3: Imaginary part of the retarded function $C_{t t}(w, q) / q^{2}$, plotted in units of $(-\chi)$, as a function of dimensionless frequency $w \equiv 3 \omega /(4 \pi T)$, for several values of dimensionless momentum $q \equiv 3 k /(4 \pi T)$. Curves from left to right correspond to $q=0.2,0.5,1.0$ (left panel), and $q=1.0,2.0,3.0,4.0$ (right panel). The dashed curves are plots of Eq. (3.37) divided by $k^{2}$.

horizon $u=1$. Then, as described in Section III B, the retarded two-point function $C_{y y}(\omega, k)$ is proportional to $\psi^{\prime}(0) / \psi(0)$, while $C_{t t}(\omega, k)$ is proportional to $\psi(0) / \psi^{\prime}(0)$.

Figure 2 shows the imaginary part of the transverse current-current correlation function, plotted in units of $(-\chi)$. At zero momentum, $\operatorname{Im} C_{y y}$ is a linear function of $w \equiv 3 \omega /(4 \pi T)$ for all $w$, as shown in the previous subsection. At large frequency, the spectral function asymptotes to $\operatorname{Im} C_{y y} \sim(-\chi) w$, regardless of the value of $q \equiv 3 k /(4 \pi T)$.

The longitudinal correlators are directly related to the conserved R-charge density, and so are more direct probes of hydrodynamic behavior, and the hydrodynamic-to-collisionless crossover. Figure 3 shows the imaginary part of the density-density correlation function divided by $q^{2}$. At small momentum and frequency, one clearly sees the diffusive peak, consistent with the hydrodynamic expression in Eq. (1.3)

$$
\operatorname{Im} C_{t t}(\omega, k)=D_{c} \chi \frac{-\omega k^{2}}{\omega^{2}+\left(D_{c} k^{2}\right)^{2}} \quad, \quad|\omega| \ll T \text { and } k \ll T .
$$

At large frequency, the asymptotic form of the spectral function is expected to be determined by the 'collisionless' ground state correlator. The latter was presented in Eq. (1.2), and here has the form

$$
\operatorname{Im} C_{t t}(\omega, k)=\frac{1}{g_{4 \mathrm{D}}^{2}} \operatorname{sgn}(\omega) \frac{\left(-k^{2}\right)}{\sqrt{\omega^{2}-k^{2}}} \quad, \quad|\omega|-k \gg T .
$$

Fig. 3, right, shows that this form is indeed well obeyed. Indeed, Eqs. (3.36) and (3.37) are exactly the correlators expected across a hydrodynamic-to-collisionless crossover in a generic system [67]: the prefactor of $k^{2}$ in Eq. (3.37) is required by charge conservation even at large $\omega$, while the factor of $1 / \sqrt{\omega^{2}-k^{2}}$ is set by the CFT current scaling dimension and Lorentz invariance.

In Fig. 4, we illustrate the crossover from the hydrodynamic regime to the collisionless regime. For each value of $q$ we find the value $w_{\max }$ where the function $\operatorname{Im} C_{t t}(w, q)$ reaches its maximal value, and plot the resulting function $w_{\max }(q)$. As we see on Fig. 4 , at small 


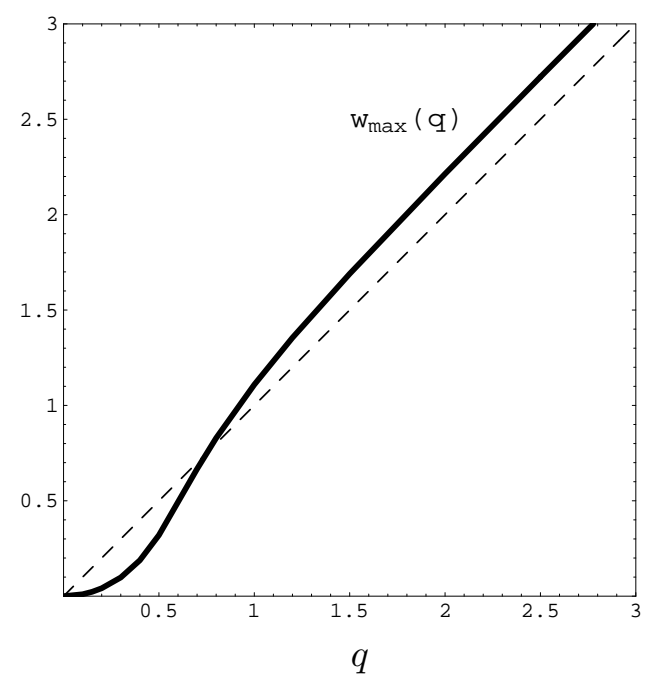

FIG. 4: The position of the peak of the spectral function in Fig. 3. The dashed line is $w=q$.

$q$ the location of the peak is $w_{\max }=q^{2}$, in accordance with hydrodynamics. At large $q$ it slowly reaches the asymptotic collisionless behavior $w_{\max }=q$.

What is unexpected, is that the two prefactors in Eqs. (3.36) and (3.37), $D_{c} \chi$ and $g_{4 \mathrm{D}}^{-2}$, happen to be equal to each other, as we saw in Eq. (3.32). We have also seen that this surprising feature is a consequence of the general functional relations in Eqs. (3.31) and (1.9). As we have discussed, such functional relations are not expected to apply to a typical $D=2+1$ CFT, but only those which enjoy special self-duality symmetries. Here the selfduality of the gauge theory on $\mathrm{AdS}_{4}$ led to the identical form of Eqs. (3.19) and (3.20) which was shown eventually to lead to Eqs. (3.31) and (1.9). In Appendix E, we consider a R-symmetry gauge field action with a non-trivial dilaton which spoils the holographic self-duality and the frequency independent conductivity. The field theory on a D2-brane in type IIA string theory is an example with such a dilaton.

\section{CONCLUSIONS}

We considered finite temperature charge transport of quantum field theories in $D=2+1$ dimensions: the easy-plane $\mathbb{C P}^{1}$ model, and the CFT living on a stack of $N$ M2-branes in M-theory (the $\mathcal{N}=8, \mathrm{SU}(N) \mathrm{SYM}$ theory). In the former theory, Abelian particle-vortex self-duality imposes a relationship (Eq. (1.8)) between different current correlators. In the latter theory, we found a strikingly similar relationship (Eq. (1.9)) between longitudinal and transverse components of the correlators of the $\mathrm{SO}(8)$ R-charge. This relationship led to a frequency-independent conductivity for the M2 worldvolume theory at zero wavevector, but hydrodynamic behavior and the hydrodynamic-collisionless crossover did appear at nonzero wavevectors. We also demonstrated that for the D2-brane theory, our argument for frequency independent conductivity fails because of a nontrivial dilaton background.

We traced the origin of the $\mathrm{SO}(8)$ charge correlation constraint of the SYM theory, and 
its frequency-independent conductivity, to an electromagnetic self-duality of the holographic theory on $\mathrm{AdS}_{4}$. Thus, the generalization of three-dimensional Abelian particle-vortex duality to non-Abelian theories becomes manifest only after a holographic extension to a fourdimensional theory. For Abelian theories, the AdS/CFT connection between particle- vortex duality in three dimensions and the $\mathrm{SL}(2, Z)$ invariance of four- dimensional Abelian gauge theories was explored earlier in $[28,57]$.

Our results for the $\mathrm{SU}(N) \mathrm{SYM}$ theory were established at large $N$. Does holographic self-duality, and the relationship ${ }^{13}$ Eq. (1.9), hold also for finite $N$ ? The fact that the large $N$ theory has hydrodynamic behavior is evidence for the "generic" nature of this limit. Furthermore, Eq. (1.9) has the same structure as Eq. (1.8), and the latter is believed to be an exact relationship, obtained without a large $N$ limit. While these facts are encouraging, establishing self-duality at finite $N$ requires looking at the full M-theory on $\mathrm{AdS}_{4}$. Its low energy limit is $\mathcal{N}=8$ supergravity [66, 68-71] (Section III considered only the $\mathrm{SO}(8)$ gauge fields of this theory), and its "generalized $\mathrm{E}_{7(7)}$ duality invariance" [69] (which appears to include EM duality) has remnants in M-theory [72].

It would be very interesting to find an Abelian field theory which obeyed a relationship as simple as Eq. (1.9), found here for the SYM theory. An unsuccessful attempt to find such a theory is described in Appendix B. The closest we could get is Eq. (1.8), obeyed by the easy-plane $\mathbb{C P}^{1}$ model [16] and its expected generalization to the SQED-2 theory with $\mathcal{N}=4$ supersymmetry [39-41]. A fundamental feature of Abelian particle-vortex duality is exchange of U(1) 'flavor' and 'topological' currents, and we have not been able to construct a theory in which these currents are equivalent to each other (which would lead to a single $K$ in Eq. (1.1)). However, non-Abelian theories can have additional symmetries which rotate different U(1) currents into each other; this was important for the simplicity of Eq. (1.9).

Finally, we would like to emphasize that the unexpected relation between the self-energies found in this paper,

$$
K^{L}(\omega, k) K^{T}(\omega, k)=\text { const },
$$

holds beyond the $\mathcal{N}=8 \mathrm{SYM}$ theory. ${ }^{14}$ It applies to the CFTs whose electromagnetic response is described by the Maxwell action (3.4) in the $3+1$ dimensional asymptotically AdS space. Thus the relation (4.1) should be viewed as another example of universality that characterizes finite-temperature response in the AdS/CFT correspondence. Previous examples of such universality include the universal value of the viscosity to entropy density ratio $\eta / s=1 / 4 \pi[77]$, and a possible universal value of the friction coefficient for a heavy

${ }^{13}$ Of course, the constant on the right-hand-side of Eq. (1.9) would have finite $N$ corrections. The issue is whether the right-hand-side remains independent of $\omega$ and $k$ for $T>0$ also at finite $N$.

${ }^{14}$ As described in Appendix C, there is a whole class of $2+1$ dimensional CFTs satisfying Eq. (4.1). For large- $N$ field theories which are dual to M-theory on $A d S_{4} \times X$, where $X$ is a seven dimensional SasakiEinstein manifold, with currents normalized as in Appendix C, the value of the constant in the right-hand side of Eq. (4.1) is $N^{3} /\left(2 \pi^{10}\right) \operatorname{Vol}(X)^{2}$. 
particle [78]. Unlike these other examples, the universal relation (4.1) applies only to $2+1$ dimensional CFTs at finite temperature. On the other hand, unlike these other examples, the universal relation (4.1) applies at arbitrary $\omega$ and $k$.

\section{Acknowledgments}

We thank M. Ernebjerg, J. Liu, D. Shih, M. Strassler, A. Strominger, C. Vafa, and A. Vishwanath for useful discussions. This research was supported by the NSF under grants PHY99-07949 (PK and CH) and DMR-0537077 (SS), and by the DOE under grants DEFG02-96ER40956 (CH) and DE-FG02-00ER41132 (DTS). C.H. thanks the organizers of the String Phenomenology workshop at the KITP, UCSB. P.K. thanks the organizers of the INT workshop "From RHIC to LHC: achievements and opportunities" at the University of Washington, and the Harvard University Physics Department, where part of this work was completed.

\section{APPENDIX A: THERMAL CORRELATORS OF CFTs IN $D=1+1$}

First, let us consider an arbitrary Lorentz-scalar observable $\mathcal{O}$ of a CFT in $D=1+1$ with scaling dimension $h$. Then, at $T=0$, its two-point correlator in Euclidean space is

$$
\left.C_{\mathcal{O}}(\tau, x)\right|_{T=0} \sim \frac{1}{\left(x^{2}+\tau^{2}\right)^{h}},
$$

while the corresponding correlator in momentum and imaginary frequency is $\sim\left(\omega^{2}+k^{2}\right)^{h-1}$. By the conformal map from the infinite plane to the cylinder with circumference $1 / T$, we can obtain the form of the correlation at $T>0$ :

$$
C_{\mathcal{O}}(\tau, x) \sim\left[\frac{\pi^{2} T^{2}}{\sin (\pi T(\tau-i x)) \sin (\pi T(\tau+i x))}\right]^{h} .
$$

Notice that this expression is periodic in $\tau$, with period $1 / T$. Now let us Fourier transform Eq. (A2) to momenta $k$ and Matsubara frequencies $\omega_{n}$; because of the periodicity, the $\omega_{n}$ must be integer multiples of $2 \pi T$, and the result is

$$
C_{\mathcal{O}}\left(i \omega_{n}, k\right) \sim T^{2 h-2} \frac{\Gamma(1-h)}{\Gamma(h)} \frac{\Gamma\left(\frac{h}{2}+\frac{\left|\omega_{n}\right|+i k}{4 \pi T}\right) \Gamma\left(\frac{h}{2}+\frac{\left|\omega_{n}\right|-i k}{4 \pi T}\right)}{\Gamma\left(1-\frac{h}{2}+\frac{\left|\omega_{n}\right|+i k}{4 \pi T}\right) \Gamma\left(1-\frac{h}{2}+\frac{\left|\omega_{n}\right|-i k}{4 \pi T}\right)} .
$$

Finally, we analytically continue this expression to real frequencies from the upper-half frequency plane $\left(\omega_{n}>0\right)$ with the mapping $i \omega_{n} \rightarrow \omega$ to obtain the retarded two-point correlator at $T>0$. This is a non-trivial function of $\omega$ and $k$, which describes relaxation of $\mathcal{O}$ correlations at $T>0$. See Chapter 4 of Ref. [31] for more details. 
Now let us consider the special case of a conserved current, and search for the collisionlessto-hydrodynamic crossover. In this case, $h=1$. Note that Eq. (A3) has a pole at $h=1$ with a residue which is $\omega$ and $k$ independent; this reflects a logarithmic cutoff dependence in the Fourier transform of Eq. (A2), and the finite $\omega$ and $k$ dependent contribution is obtained by subtracting the pole. However, the current is not a Lorentz scalar, so the above results do not directly apply anyway. The density correlator at $T=0$ in Euclidean space is

$$
\left.C_{t t}(\tau, x)\right|_{T=0} \sim \frac{1}{(\tau-i x)^{2}}+\frac{1}{(\tau+i x)^{2}} .
$$

In momentum and real frequency space, the Fourier transform of this is cutoff independent because of the non-zero Lorentz spin:

$$
\left.C_{t t}(\omega, k)\right|_{T=0} \sim \frac{-k^{2}}{k^{2}-\omega^{2}} .
$$

This is, of course, the generalization of Eq. (1.2) to $D=1+1$. We can obtain the $T>0$ density correlator by a conformal mapping of Eq. (A4), as was done earlier in Eq. (A2); here the corresponding expression is

$$
C_{t t}(\tau, x) \sim\left[\frac{\pi T}{\sin (\pi T(\tau-i x))}\right]^{2}+\left[\frac{\pi T}{\sin (\pi T(\tau+i x))}\right]^{2} .
$$

Finally, let us Fourier transform Eq. (A6) to momentum and Matsubara frequency space. Carrying out this transformation yields an initially surprising result. Although the real space result in Eq. (A6) depends upon temperature, the $T>0$ result in momentum and frequency space has the same form as that at $T=0$ in Eq. (A5):

$$
C_{t t}\left(i \omega_{n}, k\right) \sim \frac{-k^{2}}{k^{2}+\omega_{n}^{2}} .
$$

The inverse Fourier transforms of Eqs. (A5) and (A7) differ only because the frequency $\omega_{n}$ is discrete, while $\omega$ is continuous. So there is no hydrodynamic behavior at $T>0$, and no analog of the result in Eq. (1.3).

The physical interpretation of the absence of hydrodynamic behavior is simple. CFTs in $D=1+1$ can be holomorphically factorized, and consequently, there are no interactions or collisions between left and right movers. To obtain collisions, one has to consider the influence of formally irrelevant perturbations which can couple left and right movers. Only then will hydrodynamic behavior emerge: see Ref. [74]. In contrast, in $D=2+1$, hydrodynamics emerges already in the conformal scaling limit [29].

\section{APPENDIX B: ABELIAN DUALITY WITH ONE COMPLEX SCALAR}

Here we will make some remarks on the duality properties of theories of a single complex scalar coupled to a $\mathrm{U}(1)$ gauge field with a Chern-Simons term in $D=2+1$. Such theories 
have been studied extensively in the context of the quantum Hall effect [23-28, 73]. A $\mathcal{N}=3$ supersymmetric generalization of the theory below has been studied by Kapustin and Strassler [41] and their results are very similar to our $T=0$ results below. We will also present results for the theory without the Chern-Simons term (whose supersymmetric analog, noted in Section II, is the $\mathcal{N}=4$ SQED-1 theory [39-41]).

We consider a theory with the following action, which is essentially the single scalar version of Eq. (2.1), with an additional Chern-Simons term:

$$
\begin{aligned}
\mathcal{S}_{\mathrm{cs}}=\int d^{2} x d t & {\left[\left|\left(\partial_{\mu}-i A_{\mu}\right) z\right|^{2}+s|z|^{2}+u|z|^{4}\right.} \\
& \left.+\frac{1}{2 e^{2}}\left(\epsilon^{\mu \nu \lambda} \partial_{\nu} A_{\lambda}\right)^{2}+\frac{\alpha}{4 \pi} \epsilon^{\mu \nu \lambda} A_{\mu} \partial_{\nu} A_{\lambda}\right] .
\end{aligned}
$$

In general, this theory is not a CFT. However, as in Section II, we can imagine accessing a second-order phase transition out of a Higgs phase at a critical value of the "mass" term $s=s_{c}$; we are interested here in the duality properties of such a CFT.

First, standard methods $[20,21]$ can be used to obtain a dual version of the action $\mathcal{S}_{\mathrm{cs}}$ : we can either use the continuum arguments of Section II B, or apply Poisson summation methods to a lattice discretization [24, 25, 27]. From this we obtain a dual field theory, which has the same formal structure at long wavelengths:

$$
\begin{aligned}
\widetilde{\mathcal{S}}_{\mathrm{cS}}=\int d^{2} x d t & {\left[\left|\left(\partial_{\mu}-i \widetilde{A}_{\mu}\right) w\right|^{2}+\widetilde{s}|w|^{2}+\widetilde{u}|w|^{4}\right.} \\
& \left.+\frac{1}{2 \widetilde{e}^{2}}\left(\epsilon^{\mu \nu \lambda} \partial_{\nu} \widetilde{A}_{\lambda}\right)^{2}+\frac{\widetilde{\alpha}}{4 \pi} \epsilon^{\mu \nu \lambda} \widetilde{A}_{\mu} \partial_{\nu} \widetilde{A}_{\lambda}\right] .
\end{aligned}
$$

The similarity between $\mathcal{S}_{\mathrm{cs}}$ and $\widetilde{\mathcal{S}}_{\mathrm{cs}}$ is encouraging and suggests that we may be able to use it to define a self-dual CFT. However, we will now argue that this is not the case.

In general, the relationship of the coupling constants in $\mathcal{S}_{\mathrm{cs}}$ and $\widetilde{\mathcal{S}}_{\mathrm{cs}}$ is non-universal, and dependent upon the nature of the ultraviolet cutoff (with one exception, see below). However, there are a number of crucial constraints, which are readily apparent from the explicit transformations. From these constraints we find that there are 2 distinct sets of theories which are connected by duality:

Class A: Theories with no Chern-Simons terms: These theories have $\alpha=\widetilde{\alpha}=0$. Then the duality mappings show that we must have either $e=0$ or $\widetilde{e}=0$ but not both [20, 21]; this is because in a theory with zero electric charge, duality maps the coefficient of the matter kinetic energy (the "stiffness") to the electric charge squared of the dual theory. Without loss of generality, let us choose $e=0$. Then we may set $A_{\mu}=0$, which then defines $\mathcal{S}_{\mathrm{cs}}$ as the theory of a single scalar with a global U(1) symmetry (the XY model). The theory $\widetilde{\mathcal{S}}_{\mathrm{cs}}$ is the Abelian Higgs model which has a gauged U(1) 'symmetry'. Thus we have obtained 
the familiar duality [21] between the XY model and the Abelian Higgs model in $D=2+1$. It is evident from the distinct nature of these models that they are not self-dual [20, 21].

Class B: Theories with Chern-Simons terms: Now both $\alpha$ and $\widetilde{\alpha}$ must be non-zero, and indeed the lattice duality tranformations show that they satisfy

$$
\alpha \widetilde{\alpha}=-1
$$

and this is the only relationship between the couplings of $\mathcal{S}_{\text {cs }}$ and $\widetilde{\mathcal{S}}_{\text {cs }}$ which is universal. Furthermore, the requirement that either $e$ or $\widetilde{e}$ vanish no longer appears; in general, both are non-zero and finite. The duality also shows that it is not possible to eliminate the kinetic terms of both gauge fields i.e. it is not possible to set both $e=\infty$ and $\widetilde{e}=\infty$. Even if e.g. we eliminate the gauge kinetic term in $\mathcal{S}_{\mathrm{cs}}$ by setting $e=\infty$, then the duality yields a finite $\widetilde{e}$ because, by the particle-vortex prescription, the kinetic energy of $\widetilde{A}$ is related to the kinetic energy of the $z$ particles, and the latter is finite. Because we are searching for a self-dual theory, we need to keep both $e$ and $\widetilde{e}$ finite. The implication of a finite $e$ (or $\widetilde{e}$ ) is that the flux-attachment transformation associated with the Chern-Simons term is 'smeared out': each $z$ particle world-line has a total of $2 \pi / \alpha A_{\mu}$ flux attached, but this flux is spread out over a finite length scale determined by $e$. This smearing also means that the transformation $1 / \alpha \rightarrow 1 / \alpha+1$ does not map the theory onto itself. This transformation is the $T$ operation defined by Witten [28], who also found that $T$ did not leave the theory invariant. On the other hand, Fradkin and Kivelson [24] claimed $T$ invariance for their model, which was defined in terms of infinitely-thin particle and flux world-lines on a lattice with long- range interactions. It is unclear to us whether their model can be mapped to a local continuum action for a CFT.

Let us now consider correlators of the field theories without a Chern-Simons term, in class A. As discussed above, we choose the theory $\mathcal{S}_{\mathrm{cs}}$ to have $e=0$ and $\alpha=0$, so this describes the $\mathrm{O}(2) \varphi^{4}$ theory (the XY model). We are interested in the CFT at some critical $s=s_{c}$. The two-point correlator of the U(1) current, $C_{\mu \nu}$, of $\mathcal{S}_{\mathrm{cs}}$ obeys Eqs. $(1.1,1.4)$ with a single constant $K$, and a single set of functions $K^{L, T}(\omega, k)$. Similarly, the dual theory, $\widetilde{\mathcal{S}}_{\mathrm{cs}}$ (which has $\widetilde{e} \neq 0, \widetilde{\alpha}=0$ and is the Abelian Higgs model), has a correlator $\widetilde{C}_{\mu \nu}$, and the corresponding $\widetilde{K}$. Then the analog of the duality considerations of Section II imply that

$$
\begin{aligned}
K^{T}(\omega, k) \widetilde{K}^{L}(\omega, k) & =\frac{1}{4 \pi^{2}}, \\
K^{L}(\omega, k) \widetilde{K}^{T}(\omega, k) & =\frac{1}{4 \pi^{2}},
\end{aligned}
$$

and its $T=0$ limit $K \widetilde{K}=1 /\left(4 \pi^{2}\right)$. This theory in class $\mathrm{A}$ is not self-dual, so the above relations do not allow us to determine the conductivities $\sigma(\omega / T)$ and $\widetilde{\sigma}(\omega / T)$, and only constrain their product.

Next, we consider correlators of class B. The field theory $\mathcal{S}_{\mathrm{cs}}$ defines a CFT at some $s=s_{c}$, and we ask if this CFT can be self-dual. At $T=0$, we have to generalize the form 
of the current correlator $C_{\mu \nu}$ from Eq. (1.1) to $[24,27,28]$

$$
\left.C_{\mu \nu}(p)\right|_{T=0}=K \sqrt{p^{2}}\left(\eta_{\mu \nu}-\frac{p_{\mu} p_{\nu}}{p^{2}}\right)+H \epsilon_{\mu \nu \lambda} p^{\lambda}
$$

where $K, H$ are two real constants characterizing the CFT. Note that, at the gapless conformal fixed point, there is no simple relationship ${ }^{15}$ between the coupling constant $\alpha$ and the constant $H$, although a theory in class $\mathrm{B}$ is expected to have a non-zero $H$. At $T>0$, the generalization of Eq. (1.4) is

$$
C_{\mu \nu}(\omega, \boldsymbol{k})=\sqrt{p^{2}}\left(P_{\mu \nu}^{T} K^{T}(\omega, k)+P_{\mu \nu}^{L} K^{L}(\omega, k)\right)+H(\omega, k) \epsilon_{\mu \nu \lambda} p^{\lambda},
$$

with 3 distinct functions of $\omega / T$ and $k / T$ on the right hand side; note that even the Hall conductivity (equal to $H(\omega, 0)$ ) is a function of $\omega / T$ [30]. Similarly, we can also consider the dual-correlator $\widetilde{C}_{\mu \nu}$ of the theory (B2) and define a corresponding set of parameters $\widetilde{K}$ and $\widetilde{H}$. The analog $[23-25,27,28]$ of the arguments in Section II shows the following exact relationship between these parameters at $T=0$ :

$$
(H+i K)(\widetilde{H}+i \widetilde{K})=-\frac{1}{4 \pi^{2}} .
$$

The real and imaginary parts of Eq. (B7) generalize the $T=0$ limit of Eq. (B4) to class B. For $T>0$, we have

$$
\widetilde{K}^{T}(\omega, k)=\frac{K^{T}(\omega, k)}{D(\omega, k)} ; \quad \widetilde{K}^{L}(\omega, k)=\frac{K^{L}(\omega, k)}{D(\omega, k)} ; \quad \tilde{H}(\omega, k)=-\frac{H(\omega, k)}{D(\omega, k)},
$$

with

$$
D(\omega, k) \equiv 4 \pi^{2}\left(K^{T}(\omega, k) K^{L}(\omega, k)+H^{2}(\omega, k)\right) .
$$

Note that Eqs. (B8) reduce to Eqs. (B4) when $H=0$, and to Eq. (B7) at $T=0$.

For the class B model to be self-dual, we clearly need $\widetilde{K}=K$ and $\widetilde{H}=H$. From Eq. (B7) we observe that this is only possible for $K=1 /(2 \pi)$ and $H=0$. However, a model with $H=0$, which surely requires $\alpha=0$, is not in class B. It is in class A, and we argued earlier that a class A model could not be self-dual.

To conclude, although the model $\mathcal{S}_{\mathrm{cs}}$, and its dual $\widetilde{\mathcal{S}}_{\mathrm{cs}}$, define interesting CFTs, with their correlators obeying Eqs. (B4,B7,B8), we have shown that such CFTs cannot be self-dual. This conclusion is in accord with those of Kapustin and Strassler [41] and Witten [28] on related models.

\footnotetext{
15 The one-loop expression for $H$ obtained from $\mathcal{S}_{\mathrm{cs}}$ is exact as long as $s \neq s_{c}$, but the CFT at $s=s_{c}$ has corrections at all orders [30, 73].
} 


\section{APPENDIX C: NORMALIZATION OF GAUGE FIELD ACTION ON AdS}

The R-symmetry gauge field can be thought of as arising from Kaluza-Klein reduction of an 11 dimensional supergravity solution on a regular positive curvature Sasaki-Einstein manifold $X$ of real dimension seven. The size of the gauge group is determined by the isometry group of $X$. For instance, when $X=S^{7}$, the group is $\mathrm{SO}(8)$. By definition, SasakiEinstein manifolds have at least one $\mathrm{U}(1)$ isometry. In this section, we normalize the $\mathrm{U}(1)$ R-symmetry gauge field action in terms of the eleven dimensional gravitational coupling using results of Ref. [75]. Although the identification of this gauge field as a combination of metric and $F_{4}$ form perturbations in $D=11$ supergravity predates Ref. [75] (see [76]), Ref. [75] provides a convenient starting point for considering issues of normalization. The normalization is not sensitive to temperature, and hence it is convenient to work here at $T=0$.

To first order, the vector potential $A$ perturbs the eleven dimensional metric as follows:

$$
d s^{2}=\frac{r^{2}}{L^{2}} \eta_{\alpha \beta} d x^{\alpha} d x^{\beta}+L^{2} \frac{d r^{2}}{r^{2}}+4 L^{2} d s_{X}^{2}
$$

where

$$
d s_{X}^{2}=\left(\frac{q}{4}\right)^{2}\left(d \psi+\frac{4}{q} \sigma+\frac{2}{q} A\right)^{2}+h_{a \bar{b}} d z^{a} d \bar{z}^{b} .
$$

The Minkowski tensor $\eta_{\alpha \beta}$ runs over the three coordinates $x^{0}, x^{1}$, and $x^{2}$. Together the coordinates $x^{i}$ and $r$ give four dimensional anti-de Sitter space with radius of curvature $L .{ }^{16}$ Here $h_{a \bar{b}}$ is a Kähler-Einstein metric on a complex three dimensional manifold we will call $V$. Setting $A=0, X$ would be a $\mathrm{U}(1)$ fibration over the three-fold, giving rise to a real seven-dimensional Sasaki-Einstein manifold. The one form $\sigma$ is constructed such that $d \sigma=2 \omega$ where $\omega$ is the $\mathrm{K}$ ähler form on $V$. With the angle $\psi$ constrained to lie between 0 and $2 \pi$, the integer $q$ obeys the relation $\omega=\pi q c_{1} / 4$ where $c_{1}$ is the first chern class of the $\mathrm{U}(1)$ fibration. In general $q=1$, but in certain cases where $c_{1}(V)$ is divisible, $q$ may be more. For instance, in the case of $S^{7}, X$ is a $\mathrm{U}(1)$ fibration over $\mathbb{C P}^{3}$ and $q=4$. In [75], the relation between $\psi$ and $A$ was fixed by setting the R-charge of a holomorphic four-form associated to the cone over $X$ to two. This four-form has a dual field theory interpretation as a superpotential. The relation between $A$ and $\psi$ fixes the normalization of the gauge field action.

In addition to this perturbed metric, the RR four form $F_{4}$ is also perturbed by $A$ :

$$
F_{4}=\frac{3 r^{2}}{L^{3}} d^{3} x \wedge d r-4 L^{3}\left(\star_{4} d A\right) \wedge \omega
$$

Here $d^{3} x=d x^{0} \wedge d x^{1} \wedge d x^{2}$, and $\star_{4}$ is the Hodge dual in the $\mathrm{AdS}_{4}$ directions only. With $A=0, F_{4}$ can be thought of as the electric flux from a stack of M2-branes spanning the $x^{i}$ coordinates.

16 The relation to $R$ in the body of the paper is $2 L=R$. 
With these formulae for $F_{4}$ and $d s^{2}$ in hand, we can normalize the gauge field. The 11 dimensional supergravity action is

$$
\frac{1}{2 \kappa^{2}} \int d^{11} x \sqrt{-g} R-\frac{1}{4 \kappa^{2}} \int\left(F_{4} \wedge \star F_{4}+\frac{1}{3} A_{3} \wedge F_{4} \wedge F_{4}\right) .
$$

The first two terms both give contributions to $|F|^{2}$, where $F=d A$. In particular, in making $A$ nonzero, the Ricci scalar becomes

$$
R=\tilde{R}-\frac{L^{2}}{4}|F|^{2}+\frac{21}{2 L^{2}}
$$

where $|F|^{2}=F^{A B} F_{A B}$ and $\tilde{R}$ is the scalar curvature in the $A d S_{4}$ directions. Meanwhile, the four form produces a term of the form

$$
F_{4} \wedge \star F_{4}=-\left(\frac{9}{L^{2}}+\frac{3}{2} L^{2}|F|^{2}\right) \sqrt{-g} d^{11} x .
$$

We cannot simply reduce the eleven dimensional action to an effective four dimensional action as can be seen from the form of $R$ and $\left|F_{4}\right|^{2}$. Combining (C5) and (C6) in (C4) leads to a Maxwell term $|F|^{2}$ of the wrong sign. The reason Kaluza-Klein reduction does not commute with computing the equations of motion is related to the fact that the Bianchi identity $d F_{4}=0$ imposes the equation of motion $d \star F=0$ on the gauge field.

Instead, we must reduce the eleven dimensional equations of motion and from the effective four dimensional equations of motion reconstruct a four dimensional action. Along with Maxwell's equations for $F$, the eleven dimensional equations of motion reduce to

$$
R_{M N}=2 L^{2}\left(F_{M}^{P} F_{N P}-\frac{1}{4} g_{M N}|F|^{2}\right)-\frac{3}{L^{2}} g_{M N},
$$

which can be obtained from the four dimensional action

$$
S_{\text {eff }}=\frac{1}{2 \kappa_{4}^{2}} \int d^{4} x \sqrt{-g}\left(\tilde{R}-L^{2} F_{M N} F^{M N}+\frac{6}{L^{2}}\right) .
$$

Assuming that the four and eleven dimensional gravitational couplings are related by the volume of the compact manifold $X$,

$$
\frac{1}{2 \kappa_{4}^{2}}=\frac{(2 L)^{7} \operatorname{Vol}(X)}{2 \kappa^{2}}
$$

and using the standard normalization for $\kappa(3.3)$, we find that the action for the gauge field becomes

$$
-\frac{\sqrt{2} N^{3 / 2}}{2^{3} \pi^{5}} \operatorname{Vol}(X) \int d^{4} x \sqrt{-g_{4}}|F|^{2} .
$$

The volume of a seven sphere is $\operatorname{Vol}\left(S^{7}\right)=\pi^{4} / 3$. 
While in the case of more highly symmetric spaces, the R-symmetry gauge field transforms under a larger group, based on the underlying Sasaki-Einstein structure, this U(1) subgroup is in some sense the most geometrically natural.

We conclude this section by explaining, for the case of $S^{7}$, which $U(1)$ subgroup of $S O(8)$ we have extracted. Earlier, we stated that the $U(1)$ is normalized in reference to a holomorphic four-form on the cone over the Sasaki-Einstein space. For $S^{7}$, the cone is $\mathbb{C}^{4}$, and the four-form $\Omega=d X_{1} \wedge d X_{2} \wedge d X_{3} \wedge d X_{4}$ where the $X_{a}$ are complex coordinates on $\mathbb{C}^{4}$. Giving $\Omega$ R-charge two means each $X_{a}$ will have R-charge one half and will transform under the $U(1)$ group action as $X_{a} \rightarrow e^{i \alpha / 2} X_{a}$ for some phase angle $\alpha$ which runs from zero to $2 \pi$.

The Lie algebra for $S O(8)$ has four generators $\lambda_{a}$ in its Cartan sub-algebra which we can choose to act on the $X_{a}$ as $\exp \left(i \alpha \lambda_{a}\right)\left(X_{b}\right)=\delta_{a b} e^{i \alpha / 2} X_{a}$. With this normalization, $\operatorname{tr} \lambda_{a} \lambda_{b}=\frac{1}{2} \delta_{a b}$. Comparing with the action of our special $U(1)$ subgroup, we see that our $U(1)$ Lie algebra element $\lambda$ is a sum of the $\lambda_{a}: \lambda=\sum_{a} \lambda_{a}$. Thus, $\operatorname{tr} \lambda^{2}=2$.

\section{APPENDIX D: ANALYTIC SOLUTION}

At zero momentum, the mode equation (3.19) for $M(u) \equiv M_{y y}(u)$ takes the form

$$
f(u) \partial_{u}\left[f(u) \partial_{u} M(u)\right]+w^{2} M(u)=0,
$$

with $f(u)=1-u^{3}$. By introducing a new coordinate $z=\int_{0}^{u} d \tilde{u} / f(\tilde{u})$, the equation simplifies,

$$
\partial_{z}^{2} M(z)+w^{2} M(z)=0
$$

with the boundary condition $M(z=0)=1$ at the boundary, and the outgoing condition at the horizon $z=\infty$. The solution is

$$
M(z)=e^{i w z} .
$$

That it corresponds to outgoing waves can be seen from the fact that in the function $e^{-i \omega(t-z)}$ the wave front moves toward larger $z$, i.e. closer to the horizon as $t$ increases. Therefore we find

$$
M(u)=\exp \left[i w \int_{0}^{u} \frac{d \tilde{u}}{f(\tilde{u})}\right]
$$

The leading asymptotics for $u$ near zero is $M(u)=1+i w u$. From the AdS/CFT prescription (3.14) we immediately find

$$
C_{y y}(w, 0)=\Pi^{T}(w, 0)=\Pi^{L}(w, 0)=-i \chi w
$$

This agrees with the result for conductivity in section III C, as it should. 


\section{APPENDIX E: GAUGE FIELD WITH A DILATON}

Consider a $\mathrm{U}(1)$ gauge field on a four dimensional manifold $M$ with an action of the form

$$
S=-\frac{1}{2 g_{4 \mathrm{D}}^{2}} \int_{M} e^{-2 \phi} F \wedge \star F .
$$

There are a number of interesting $2+1$ dimensional field theories which have a dual $\mathrm{R}$ symmetry gauge field of this type - for example the M2-brane theory at finite R-charge chemical potential and the D2-branes in type IIA string theory. Here $F$ is the two-form gauge field, $\phi$ a dilaton like scalar, and $g_{4 \mathrm{D}}$ the coupling. The Maxwell equations can be written elegantly as $d F=0$ and $d \star e^{-2 \phi} F=0$. There is an equivalent S-dual theory where the roles of $F$ and $\widetilde{F} \equiv \star e^{-2 \phi} F$ are interchanged and we send $g_{4 \mathrm{D}} e^{\phi} \rightarrow \widetilde{g}_{4 \mathrm{D}} e^{\widetilde{\phi}} \equiv 1 /\left(g_{4 \mathrm{D}} e^{\phi}\right)$ :

$$
S=-\frac{1}{2 \widetilde{g}_{4 \mathrm{D}}^{2}} \int_{M} e^{2 \phi} \widetilde{F} \wedge \star \widetilde{F}
$$

The point we would like to emphasize is that when $\phi$ is a constant, the theory is almost self-dual in the sense that the equations of motion for $F$ and $\widetilde{F}$ are identical. When $\phi$ is not a constant, the equations of motion for $F$ and $\widetilde{F}$ are identical up to sending $\phi \rightarrow-\phi$.

We would like to investigate the consequences of this duality in the context of the AdS/CFT correspondence where this gauge field is interpreted as a bulk field corresponding to some global U(1) symmetry on a $2+1$ dimensional boundary theory. To this end, we assume the metric takes the diagonal form

$$
d s^{2}=-g_{t t}(u) d t^{2}+d u^{2}+g_{x x}(u)\left(d x^{2}+d y^{2}\right)
$$

where the metric components are only radially dependent on a coordinate we call $u$. By diffeomorphism invariance, we can always set $g_{u u}=1$. The boundary is taken to be located at $u=0$ and the interior for $u>0$ with a horizon at $u=u_{h}>0$. We will assume that as $u \rightarrow 0,-g_{t t} \sim g_{x x} \sim c^{2} / u^{\alpha}$ where $\alpha>-2$.

We will calculate two-point functions of the $\mathrm{U}(1)$ current $J$ corresponding to this global symmetry. Introducing a vector potential $F=d A$, the retarded two-point function can be found using the method described in Sec. III B. Namely, one looks for the solution to the field equation for $A_{\nu}$ of the form vector potential of the form

$$
A_{\nu}(x, t, u)=e^{i p \cdot x} M_{\nu}^{\mu}(p, u) A_{\mu}^{0}(p)
$$

where $M_{\nu}{ }^{\mu}(p, u)$ satisfies the radial component of the equation of motion for $A_{\nu}$. Furthermore, $M_{\nu}{ }^{\mu}(p, 0)=\delta_{\nu}^{\mu}$ and $M_{\nu}{ }^{\mu}$ satisfies outgoing boundary condition at the horizon $u=u_{h}$. If the kinetic term for $A_{\mu}$ can be written as

$$
-\frac{1}{2 g_{4 \mathrm{D}}^{2}} \int d u d^{3} x G(u)\left(A_{\mu}^{\prime}\right)^{2}
$$


then

$$
C^{\mu \nu}(k)=-\frac{1}{g_{4 \mathrm{D}}^{2}} \lim _{u \rightarrow 0} G(u) \frac{\partial}{\partial u} M^{\mu \nu}(p, u) .
$$

In particular, we take $A^{\mu}(x, t, u)$ to satisfy the equation of motion

$$
\partial_{A}\left[e^{-2 \phi} \sqrt{-g} g^{A B} g^{C D}\left(A_{B, D}-A_{D, B}\right)\right]=0
$$

and fix a radial gauge $A_{u}=0$. We also choose $p^{\mu}=(\omega, k, 0)$. In this gauge, the equation of motion for $A_{y}$ becomes

$$
\partial_{u}\left[e^{-2 \phi} \sqrt{-g} g^{x x} A_{y}^{\prime}\right]-k^{2} \sqrt{-g}\left(g^{x x}\right)^{2} e^{-2 \phi} A_{y}-\omega^{2} \sqrt{-g} g^{x x} g^{t t} e^{-2 \phi} A_{y}=0 .
$$

Because of the constraint on $\alpha$, the near boundary behavior of $A_{y}(u \sim 0)$ is governed by an expansion of the form

$$
M^{y y}(p, u)=(1+\mathcal{O}(u))+u^{1+\alpha / 2} \mathcal{B}(1+\mathcal{O}(u)) .
$$

In the case where $\alpha$ is an even integer, the two series will overlap, leading to logarithmic terms in the first series, which complicate the story but should not alter it in any fundamental way. The constant $\mathcal{B}$ is a complicated function of $\omega$ and $k$ which is determined by fixing outgoing boundary conditions at the horizon $u=u_{h}$. For $A_{y}$, the function $G(u)$ in (E5) is $\sqrt{-g} g^{x x}$ which near the boundary scales as $c u^{-\alpha / 2}$ where $c$ depends on the precise form of our metric. By absorbing $\phi(0)$ into the value of $g_{4 \mathrm{D}}$, we can choose $\phi(0)=0$. From this expansion and the form of $G(u)$, clearly

$$
C^{y y}=\frac{1}{g_{4 \mathrm{D}}^{2}}(1+\alpha / 2) c \mathcal{B} .
$$

In our gauge, $A_{y}$ can be reinterpreted as a radial magnetic field, $B_{u}=F_{x y}=-i k A_{y}$. By electric-magnetic duality, replacing $\phi$ with $-\phi$, the equation of motion for $B_{u}(\mathrm{E} 8)$ must be the same as the equation of motion for $E_{u} \equiv-\left(\star e^{-2 \phi} F\right)_{x y}=\sqrt{-g} g^{t t} e^{-2 \phi} A_{t}^{\prime}$ :

$$
\partial_{u}\left[e^{2 \phi} \sqrt{-g} g^{x x} E_{u}^{\prime}\right]-k^{2} \sqrt{-g}\left(g^{x x}\right)^{2} e^{2 \phi} E_{u}-\omega^{2} \sqrt{-g} g^{x x} g^{t t} e^{2 \phi} E_{u}=0
$$

We thus know that $E_{u}$ has the near boundary expansion

$$
E_{u}=E_{u}^{0} e^{i p \cdot x}\left[(1+\mathcal{O}(u))+u^{1+\alpha / 2} \widetilde{\mathcal{B}}(1+\mathcal{O}(u))\right] .
$$

The tilde over $\mathcal{B}$ indicates it was derived from (E8) having replaced $\phi$ with $-\phi$. In the case $\phi=$ const, $\mathcal{B}=\widetilde{\mathcal{B}}$. We now use Gauss's law to constrain the boundary behavior of $E_{u}^{0}$. The equation of motion following from taking the index $C=t$ in (E7) is

$$
\left(\sqrt{-g} g^{t t} e^{-2 \phi} A_{t}^{\prime}\right)^{\prime}-k \sqrt{-g} g^{t t} g^{x x} e^{-2 \phi}\left(\omega A_{x}+k A_{t}\right)=0 .
$$

From the near boundary behavior, we find that

$$
E_{u}^{0}(1+\alpha / 2) \widetilde{\mathcal{B}}=-\frac{k}{c}\left(\omega A_{x}^{0}+k A_{t}^{0}\right)
$$


We can run a similar analysis of the component $A_{x}^{\prime}$ and construct a full boundary action. We find that

$$
\begin{aligned}
S_{b}= & -\frac{1}{2 g_{4 \mathrm{D}}^{2}} \int_{\mathbb{R}^{3}} d^{3} x\left[\mathcal{B} c(1+\alpha / 2)\left(A_{y}^{0}\right)^{2}-\right. \\
& \left.\frac{1}{\widetilde{\mathcal{B}} c(1+\alpha / 2)}\left(k^{2}\left(A_{t}^{0}\right)^{2}+\omega^{2}\left(A_{x}^{0}\right)^{2}+\omega k A_{t}^{0} A_{x}^{0}\right)\right] .
\end{aligned}
$$

From this normalization, we conclude that the remaining two point functions are

$$
\begin{aligned}
C^{t t} & =-\frac{1}{g_{4 \mathrm{D}}^{2}} \frac{k^{2}}{c(1+\alpha / 2) \widetilde{\mathcal{B}}}, \\
C^{x t} & =-\frac{1}{g_{4 \mathrm{D}}^{2}} \frac{k \omega}{c(1+\alpha / 2) \widetilde{\mathcal{B}}}, \\
C^{x x} & =-\frac{1}{g_{4 \mathrm{D}}^{2}} \frac{\omega^{2}}{c(1+\alpha / 2) \widetilde{\mathcal{B}}} .
\end{aligned}
$$

In the special case where $\phi$ is a constant and hence $\widetilde{\mathcal{B}}=\mathcal{B}$, we find that

$$
C^{t t} C^{y y}=-\frac{1}{g_{4 \mathrm{D}}^{4}} k^{2} ; \quad C^{x t} C^{y y}=-\frac{1}{g_{4 \mathrm{D}}^{4}} k \omega ; \quad C^{x x} C^{y y}=-\frac{1}{g_{4 \mathrm{D}}^{4}} \omega^{2} .
$$

[1] R. W. Crane, N. P. Armitage, A. Johansson, G. Sambandamurthy, D. Shahar, and G. Grüner, "Survival of Superconducting Correlations Across the 2D Superconductor-Insulator Transition: A finite frequency study," cond-mat/0604107.

[2] M. A. Steiner, G. Boebinger, and A. Kapitulnik, "Possible Field-Tuned SuperconductorInsulator Transition in High- $T_{c}$ Superconductors: Implications for Pairing at High Magnetic Fields," Phys. Rev. Lett. 94, 107008 (2005), cond-mat/0406232.

[3] G. Sambandamurthy, L. W. Engel, A. Johansson, E. Peled, and D. Shahar, "Experimental Evidence for a Collective Insulating State in Two-Dimensional Superconductors," Phys. Rev. Lett. 94, 017003 (2005), cond-mat/0403480.

[4] D. Shahar, D. C. Tsui, M. Shayegan, E. Shimshoni, and S. L. Sondhi, "A Different View of the Quantum Hall Plateau-to-Plateau Transitions," Phys. Rev. Lett. 79, 479 (1997), cond-mat/9611011.

[5] L. W. Engel, D. Shahar, Ç. Kurdak, and D. C. Tsui, "Microwave frequency dependence of integer quantum Hall effect: Evidence for finite-frequency scaling," Phys. Rev. Lett. 71, 2638 (1993).

[6] M. B. Stone, C. Broholm, D. H. Reich, P. Schiffer, O. Tchernyshyov, P. Vorderwisch, and N. Harrison, "Field-driven phase transitions in a quasi-two-dimensional quantum antiferromagnet," New J. Phys. 9, 31 (2007), cond-mat/0611463. 
[7] E. Demler, S. Sachdev and Y. Zhang, "Spin ordering quantum transitions of superconductors in a magnetic field," Phys. Rev. Lett. 87, 067202 (2001), cond-mat/0103192.

[8] B. Khaykovich, S. Wakimoto, R. J. Birgeneau, M. A. Kastner, and Y. S. Lee, P. Smeibidl, P. Vorderwisch, and K. Yamada, "Field-induced transition between magnetically disordered and ordered phases in underdoped $\mathrm{La}_{2-x} \mathrm{Sr}_{x} \mathrm{CuO}_{4}$," Phys. Rev. B 71, 220508 (2005), cond-mat/0411355.

[9] M. P. A. Fisher, P. B. Weichman, G. Grinstein, and D. S. Fisher, "Boson localization and the superfluid-insulator transition," Phys. Rev. B 40, 546 (1989).

[10] M. Greiner, O. Mandel, T. Esslinger, T. W. Hänsch, and I. Bloch, "Quantum phase transition from a superfluid to a Mott insulator in a gas of ultracold atoms," Nature 415, 39 (2002).

[11] I. B. Spielman, W. D. Phillips, and J. V. Porto, "Mott-Insulator Transition in a TwoDimensional Atomic Bose Gas," Phys. Rev. Lett. 98, 080404 (2007), cond-mat/0606216.

[12] L. Wang, K. S. D. Beach, and A. W. Sandvik, "High-precision finite-size scaling analysis of the quantum-critical point of $S=1 / 2$ Heisenberg antiferromagnetic bilayers," Phys. Rev. B 73, 014431 (2006), cond-mat/0509747.

[13] M. Matsumoto, C. Yasuda, S. Todo, and H. Takayama, "Ground-state phase diagram of quantum Heisenberg antiferromagnets on the anisotropic dimerized square lattice," Phys. Rev. B 65, 014407 (2002), cond-mat/0107115.

[14] T. Senthil, A. Vishwanath, L. Balents, S. Sachdev, and M. P. A. Fisher, "Deconfined quantum critical points," Science 303, 1490 (2004), cond-mat/0311326 ; T. Senthil, L. Balents, S. Sachdev, A. Vishwanath, and M. P. A. Fisher, "Quantum criticality beyond the LandauGinzburg-Wilson paradigm," Phys. Rev. B 70, 144407 (2004), cond-mat/0312617.

[15] A. W. Sandvik, "Evidence for deconfined quantum criticality in a two-dimensional Heisenberg model with four-spin interactions," cond-mat/0611343.

[16] O. I. Motrunich and A. Vishwanath, "Emergent photons and new transitions in the $\mathrm{O}(3)$ sigma model with hedgehog suppression," Phys. Rev. B 70, 075104 (2004), cond-mat/0311222.

[17] M. P. A. Fisher, G. Grinstein, and S. M. Girvin, "Presence of quantum diffusion in two dimensions: Universal resistance at the superconductor-insulator transition," Phys. Rev. Lett. 64, 587 (1990).

[18] M. P. A. Fisher, "Quantum phase transitions in disordered two-dimensional superconductors," Phys. Rev. Lett. 65, 923 (1990).

[19] X.-G. Wen and A. Zee, "Universal conductance at the superconductor-insulator transition," Int. J. Mod. Phys. B 4, 437 (1990).

[20] M. E. Peskin, "Mandelstam-'t Hooft duality in abelian lattice models," Annals of Physics, 113, 122 (1978).

[21] C. Dasgupta and B. I. Halperin, "Phase Transition in a Lattice Model of Superconductivity," Phys. Rev. Lett. 47, 1556 (1981).

[22] M. P. A. Fisher and D. H. Lee, "Correspondence between two-dimensional bosons and a bulk 
superconductor in a magnetic field," Phys. Rev. B 39, 2756 (1989).

[23] D.-H. Lee and M. P. A. Fisher, "Anyon Superconductivity and the Fractional Quantum Hall Effect", Phys. Rev. Lett. 63, 1442 (1989).

[24] E. Fradkin and S. Kivelson, "Modular invariance, self-duality and the phase transition between quantum Hall plateaus," Nucl. Phys. B 474, 543 (1996), cond-mat/9603156.

[25] L. P. Pryadko and S.-C. Zhang, "Duality and universality for the Chern-Simons bosons", Phys. Rev. B 54, 4953 (1996), cond-mat/9511140.

[26] E. Shimshoni, S. L. Sondhi, and D. Shahar, "Duality near quantum Hall transitions," Phys. Rev. B 55, 13730 (1997), cond-mat/9610102.

[27] C. P. Burgess and B. P. Dolan, "Particle-vortex duality and the modular group: Applications to the quantum Hall effect and other 2-D systems," Phys. Rev. B 63, 155309 (2001), hep-th/0010246.

[28] E. Witten, "SL $(2, \mathrm{Z})$ action on three-dimensional conformal field theories with Abelian symmetry," hep-th/0307041.

[29] K. Damle and S. Sachdev, "Non-zero temperature transport near quantum critical points," Phys. Rev. B 56, 8714 (1997), cond-mat/9705206.

[30] S. Sachdev, "Non-zero temperature transport near fractional quantum Hall critical points," Phys. Rev. B 57, 7157 (1998), cond-mat/9709243.

[31] S. Sachdev, Quantum Phase Transitions, Cambridge University Press, Cambridge (1999).

[32] S. Sachdev and J. Ye, "Universal quantum critical dynamics of two-dimensional antiferromagnets," Phys. Rev. Lett. 69, 2411 (1992), cond-mat/9204001.

[33] S. Hod, "Universal Bound on Dynamical Relaxation Times and Black-Hole Quasinormal Ringing," gr-qc/0611004.

[34] A. V. Chubukov, S. Sachdev, and J. Ye, "Theory of two-dimensional quantum antiferromagnets with a nearly-critical ground state," Phys. Rev. B 49, 11919 (1994), cond-mat/9304046.

[35] J. Šmakov and E. Sørensen, "Universal Scaling of the Conductivity at the Superfluid-Insulator Phase Transition," Phys. Rev. Lett. 95, 180603 (2005), cond-mat/0509671.

[36] A. G. Green and S. L. Sondhi, "Nonlinear Quantum Critical Transport and the Schwinger Mechanism for a Superfluid-Mott-Insulator Transition of Bosons," Phys. Rev. Lett. 95, 267001 (2005), cond-mat/0501758.

[37] A. G. Green, J. E. Moore, S. L. Sondhi, and A. Vishwanath, "Current fluctuations near to the $2 \mathrm{D}$ superconductor-insulator quantum critical point," cond-mat/0605615.

[38] M. J. Bhaseen, A. G. Green, and S. L. Sondhi, "Magnetothermoelectric Response at a Superfluid-Mott Insulator Transition," cond-mat/0610687.

[39] K. A. Intriligator and N. Seiberg, "Mirror symmetry in three dimensional gauge theories," Phys. Lett. B 387, 513 (1996), hep-th/9607207.

[40] O. Aharony, A. Hanany, K. A. Intriligator, N. Seiberg and M. J. Strassler, "Aspects of $\mathcal{N}=2$ supersymmetric gauge theories in three dimensions," Nucl. Phys. B 499, 67 (1997), 
hep-th/9703110.

[41] A. Kapustin and M. J. Strassler, "On mirror symmetry in three dimensional Abelian gauge theories," JHEP 9904, 021 (1999), hep-th/9902033.

[42] L. Balents, L. Bartosch, A. Burkov, S. Sachdev, and K. Sengupta, "Putting competing orders in their place near the Mott transition," Phys. Rev. B 71, 144508 (2005), cond-mat/0408329.

[43] N. Seiberg, "Notes on theories with 16 supercharges," Nucl. Phys. Proc. Suppl. 67, 158 (1998), hep-th/9705117.

[44] S. Sethi and L. Susskind, "Rotational invariance in the M(atrix) formulation of type IIB theory," Phys. Lett. B 400, 265 (1997), hep-th/9702101.

[45] N. Itzhaki, J. M. Maldacena, J. Sonnenschein and S. Yankielowicz, "Supergravity and the large $N$ limit of theories with sixteen supercharges," Phys. Rev. D 58, 046004 (1998), hep-th/9802042.

[46] See section 6.1 of the review O. Aharony, S. Gubser, J. Maldacena, H. Ooguri and Y. Oz, "Large N field theories, string theory and gravity," Phys. Rept. 323, 183 (2000), hep-th/9905111.

[47] D. T. Son and A. O. Starinets, "Minkowski-space correlators in AdS/CFT correspondence: Recipe and applications," JHEP 0209, 042 (2002), hep-th/0205051.

[48] C. P. Herzog and D. T. Son, "Schwinger-Keldysh propagators from AdS/CFT correspondence," JHEP 0303, 046 (2003), hep-th/0212072.

[49] C. P. Herzog, "The hydrodynamics of M-theory", JHEP 0212, 026 (2002), hep-th/0210126.

[50] G. Policastro, D. T. Son and A. O. Starinets, "From AdS/CFT correspondence to hydrodynamics," JHEP 0209, 043 (2002), hep-th/0205052.

[51] P. Kovtun, D. T. Son and A. O. Starinets, "Holography and hydrodynamics: Diffusion on stretched horizons," JHEP 0310, 064 (2003), hep-th/0309213.

[52] A. Buchel, “N = 2* hydrodynamics," Nucl. Phys. B 708, 451 (2005), hep-th/0406200.

[53] P. Benincasa and A. Buchel, "Hydrodynamics of Sakai-Sugimoto model in the quenched approximation," Phys. Lett. B 640, 108 (2006), hep-th/0605076.

[54] D. Mateos, R. C. Myers and R. M. Thomson, "Holographic viscosity of fundamental matter," hep-th/0610184.

[55] S. Caron-Huot, P. Kovtun, G. D. Moore, A. Starinets and L. G. Yaffe, "Photon and dilepton production in supersymmetric Yang-Mills plasma," JHEP 0612, 015 (2006), hep-th/0607237.

[56] S. Sethi, "A relation between $\mathcal{N}=8$ gauge theories in three dimensions," JHEP 9811, 003 (1998), hep-th/9809162.

[57] R. G. Leigh and A. C. Petkou, "SL $(2, Z)$ action on three-dimensional CFTs and holography," JHEP 0312, 020 (2003), hep-th/0309177.

[58] E. Babaev, "Vortices with Fractional Flux in Two-Gap Superconductors and in Extended Faddeev Model ," Phys. Rev. Lett. 89, 067001 (2002), cond-mat/0111192.

[59] A. M. Polyakov, "Compact gauge fields and the infrared catastrophe," Phys. Lett. B 59, 82 
(1975).

[60] S. Sachdev, "Quantum phases and phase transitions of Mott insulators," in Quantum magnetism, U. Schollwock, J. Richter, D. J. J. Farnell and R. A. Bishop eds, Lecture Notes in Physics 645, Springer, Berlin (2004), cond-mat/0401041.

[61] A. Kuklov, N. Prokof'ev, B. Svistunov, and M. Troyer, "Deconfined criticality, runaway flow in the two-component scalar electrodynamics and weak first-order superfluid-solid transitions," Annals of Physics 321, 1602 (2006), cond-mat/0602466.

[62] E. D'Hoker and D. Z. Freedman, "Supersymmetric gauge theories and the AdS/CFT correspondence," in Strings, Branes And Extra Dimensions: Tasi 2001, S. S. Gubser and J. D. Lykken Eds., World Scientific, Singapore (2004), hep-th/0201253.

[63] E. Witten, "Anti-de Sitter space, thermal phase transition, and confinement in gauge theories," Adv. Theor. Math. Phys. 2, 505 (1998), hep-th/9803131.

[64] C. P. Herzog, "A holographic prediction of the deconfinement temperature," Phys. Rev. Lett. 98, 091601 (2007) hep-th/0608151.

[65] G. T. Horowitz and A. Strominger, "Black strings and P-branes," Nucl. Phys. B 360, 197 (1991).

[66] M. J. Duff and J. T. Liu, "Anti-de Sitter Black Holes in Gauged $\mathcal{N}=8$ Supergravity," Nucl. Phys. B 554, 237 (1999), hep-th/9901149.

[67] D. Forster, Hydrodynamic Fluctuations, Broken Symmetry, and Correlation Functions, Benjamin/Cummings, Reading MA (1975).

[68] E. Cremmer and B. Julia, "The SO(8) Supergravity," Nucl. Phys. B 159, 141 (1979).

[69] B. de Wit and H. Nicolai, “N $=8$ Supergravity," Nucl. Phys. B 208, 323 (1982).

[70] M. J. Duff and C. N. Pope, "Kaluza-Klein Supergravity And The Seven Sphere," Supersymmetry and Supergravity 82, Proc. trieste Conf. ed S. Ferrara, J. G. Taylor, and P. van Nieuwenhuizen, pp 183-228, World Scientific (Singapore).

[71] P. G. O. Freund, Introduction to Supersymmetry, Chapters 23-26, Cambridge University Press, Cambridge (1986).

[72] C. M. Hull and P. K. Townsend, "Unity of superstring dualities," Nucl. Phys. B 438, 109 (1995), hep-th/9410167.

[73] X. G. Wen and Y.-S. Wu, "Transitions between the quantum Hall states and insulators induced by periodic potentials," Phys. Rev. Lett. 70, 1501 (1993).

[74] T. Giamarchi, "Resistivity of a one-dimensional interacting quantum fluid," Phys. Rev. B 46, 342 (1992).

[75] D. Berenstein, C. P. Herzog and I. R. Klebanov, "Baryon spectra and AdS/CFT correspondence," JHEP 0206, 047 (2002), hep-th/0202150.

[76] M. J. Duff, C. N. Pope and N. P. Warner, "Cosmological And Coupling Constants In KaluzaKlein Supergravity," Phys. Lett. B 130, 254 (1983).

[77] P. Kovtun, D. T. Son and A. O. Starinets, "Viscosity in strongly interacting quantum field 
theories from black hole physics," Phys. Rev. Lett. 94, 111601 (2005), hep-th/0405231.

[78] C. P. Herzog, "Energy loss of heavy quarks from asymptotically AdS geometries," JHEP 0609, 032 (2006), hep-th/0605191. 